\title{
ENERGY FLUXES AND OHMIC DISSIPATION IN THE EARTH'S CORE
}

\author{
P.H. Roberts \\ Institute of Geophysics and Planetary Physics \\ University of California, Los Angeles, CA 90095, USA \\ C.A. Jones \\ School of Mathematical Sciences, University of Exeter \\ Exeter EX4 4QE, UK \\ A.R. Calderwood \\ Physics Department, University of Nevada-Las Vegas \\ Las Vegas, NV 89154, USA
}

\begin{abstract}
Simulations have shown that convection-driven geodynamo models can produce reversing magnetic fields of a similar strength to those of the geomagnetic field. These simulations suggest that the ohmic dissipation arising from the geodynamo is not due solely to the largest scale harmonics of the magnetic field, but is dominated by contributions from smaller scale components. It is argued that, in order to produce a vigorous reversing dynamo resembling the geodynamo, ohmic dissipation of between 1 and $2 \mathrm{TW}$ is required.

The energy and entropy fluxes through the core are investigated to see under what circumstances the required ohmic dissipation can be produced. The estimates for these fluxes given in Braginsky and Roberts (1995) are revised in the light recent theoretical and experimental advances in high pressure physics. In the absence of radioactive heating in the core, the available thermal and gravitational energy sources can produce more than 1 TW of ohmic dissipation provided the inner core is not significantly older than 1.2 Gyr. If the inner core is much older than this, the thermal and gravitational energy sources are released at too slow a rate to give the required amount of ohmic dissipation. Paleomagnetic evidence suggests that a geomagnetic field of approximately today's strength has been in existence for over $3 \mathrm{Gyr}$, perhaps before the inner core came into existence. Estimates of the available energy sources based on current estimates of the heat flux passing through the core-mantle boundary suggest that they may be insufficient to sustain such a field. We consider the possibility that significant radioactivity in the core could resolve this difficulty.
\end{abstract}

\section{INTRODUCTION}

Many bodies in the solar system are known to have dense central cores, examples being Earth, Venus, Mars, Mercury, Ganymede. Some of these bodies (e.g., Earth, Mercury, Ganymede) create their own magnetic fields by dynamo action in their cores; others (e.g., Venus, Mars) do not. In the case of the Earth, the principal example used in this article, there are strong indications that the core is composed of an iron-rich alloy; the cores of the other terrestrial planets and satellites are usually taken to have a similar metallic composition. The salient property of such materials (when in a melted state) is a magnetic diffusivity $\eta_{M}$ that is large compared with all other diffusivities, such as the kinematic viscosity $\nu_{M}$ and the thermal diffusivity $\kappa_{M}$, where the suffix ${ }_{M}$, standing for 'Molecular', is added to avoid confusion with the 'Turbulent' diffusivities to be introduced later, for 
which $T$ is used. The Prandtl numbers,

$$
P_{m} \equiv \nu_{M} / \eta_{M}, \quad q \equiv \kappa_{M} / \eta_{M},
$$

are therefore very small: values quoted for the Earth's core are typically $P_{m} \sim 10^{-6}$ and $q \sim 10^{-5}$.

To maintain a magnetic field successfully, the core flow speed, of order $\mathcal{V}$ (say), must be sufficently large, as measured by the magnetic Reynolds number

$$
R m \equiv \mathcal{V} \mathcal{L} / \eta_{M}
$$

where $\mathcal{L}$ is the depth of the fluid core. A characteristic value of $R m$ for a working dynamo is taken here to be 100, which by (1.1) implies that

$$
R e \equiv \mathcal{V} \mathcal{L} / \nu_{M}, \quad P e \equiv \mathcal{V} \mathcal{L} / \kappa_{M}, \quad M p \equiv \mathcal{V} \mathcal{L} / D_{M},
$$

are all very large. We have here included, with the (kinetic) Reynolds number $R e \sim 10^{8}$ and the Péclet number $P e \sim 10^{7}$, the mass Péclet number $M p \sim 10^{11}$ which is a dimensionless measure of the diffusivity, $D$, between the different chemical constituents present in the core. The predominant composition of the Earth's core is unknown, FeS, FeSi and $\mathrm{FeO}$ having all been advocated. Recent work (Price et al., presented as a poster in this meeting) indicates that a trinary alloy is more realistic. To generalize the following from 2 to 3 (or indeed many) components is easy, but adds complications without compensating enlightenment; for simplicity, we shall model the fluid as a binary alloy. The large values of $R e, P e$ and $M p$ indicate that molecular diffusion of large-scale momentum, heat and composition are totally negligible, and that these quantities must be transported predominantly by small-scale eddies which, while they may contain very little kinetic energy, are far more effective diffusers than molecules.

It is impossible now or in the foreseeable future to resolve numerically the small-scale eddies that diffuse the large-scale fields. For instance, in the case of Earth, it would be necessary to resolve length scales of the order of $\ell \sim 1 \mathrm{~km}$, in a body of fluid of depth $\mathcal{L} \sim$ $2900 \mathrm{~km}$, and to follow time-scales of order $\tau \sim 300 \mathrm{~s}$ in a system where the overturning time of large eddies is of order $300 \mathrm{yr}\left(=10^{10} \mathrm{~s}\right)$. Lacking anything better, we employ the popular two-scale approach in which the flow $\mathbf{V}$ is modeled as a superposition of a small-scale turbulent velocity $\mathbf{V}^{\prime}$ and a large-scale laminar motion $\overline{\mathbf{V}}$, so that $\mathbf{V}=\overline{\mathbf{V}}+\mathbf{V}^{\prime}$. We then follow Osborne Reynolds who, likening the nearly random motion of eddies to the nearly random motion of molecules, argued that the effect of turbulence on the transport of large-scale momentum could be well represented by replacing the molecular kinematic viscosity $\nu_{M}$ by a much greater 'turbulent viscosity', $\nu_{T}$. According to the Reynolds analogy, the flux, at position $\mathbf{x}$ and time $t$, of large-scale momentum created by the smallscale eddies depends only on the rates of strain of the large-scale velocity, $\overline{\mathbf{V}}(\mathbf{x}, t)$, at the same $\mathbf{x}$ and $t$. In other words, the analogy leads to a local description of turbulence, in which the inertial term $\overline{\mathbf{V}^{\prime} \cdot \nabla \mathbf{V}^{\prime}}$ in the equation governing $\overline{\mathbf{V}}$ is represented by $-\nu_{T} \nabla^{2} \overline{\mathbf{V}}$. This has the effect of replacing $\nu_{M}$ by $\nu_{\text {total }}=\nu_{T}+\nu_{M}$ in the equation governing $\overline{\mathbf{V}}$, but since $\nu_{T} \gg \nu_{M}$ it is acceptable to set $\nu_{\text {total }}=\nu_{T}$. 
The hugeness of $P e$ and $M p$ suggests that, in the case of the specific entropy $S$ and the composition $\xi$ (i.e., the mass fraction of the light component in the alloy), the convective fluxes $\mathbf{I}^{S}=\rho_{a} \overline{S^{\prime} \mathbf{V}^{\prime}}$ and $\mathbf{I}^{\xi}=\rho_{a} \overline{\xi^{\prime} \mathbf{V}^{\prime}}$ of large-scale entropy and composition created by the small scale motions may be replaced respectively by $-\rho_{a} \kappa_{T} \nabla \bar{S}$ and $-\rho_{a} D_{T} \nabla \bar{\xi}$ where, again, the diffusivities $\kappa_{T}$ and $D_{T}$ so greatly exceed $\kappa_{M}$ and $D_{M}$ that they can be used to replace the more precise $\kappa_{\text {total }}$ and $D_{\text {total }}$. Moreover, Braginsky and Roberts (1995) argue that, since $S$ and $\xi$ are extensive variables that are transported by turbulent eddies in the same way, $D_{T}=\kappa_{T}$.

The Reynolds analogy implicity assumes that the eddy motions, like the molecular motions, are approximately isotropic. It has been cogently argued however by Braginsky (1964) and Braginsky and Meytlis (1990) that core turbulence is far from isotropic; see also Braginsky and Roberts (1995) and St. Pierre (1996). Thus $\nu_{T}$ and $\kappa_{T}$ should be replaced by tensors. This has never been done in numerical simulations, although a different (and undesirable) anisotropy is present in many geodynamo simulations with the introduction of hyperviscosity, which enhances diffusion in the horizontal directions but not in the radial direction. This is done for purely pragmatic reasons; the anisotropy of core turbulence is almost certainly determined by the directions of the prevailing mean field, $\overline{\mathbf{B}}$, and the rotation of the system, $\boldsymbol{\Omega}$, and not by the local direction of gravity $\mathbf{g}$. In what follows we shall follow current practice by ignoring diffusive anisotropies. We shall sometimes use the terms 'macroscale' and 'microscale' instead of 'large-scale' and 'small-scale'.

A perplexing question concerns the effect of turbulence on the mean magnetic field $\overline{\mathbf{B}}$, "Does microscale induction significantly affect the macroscales?" Put another way, "Must the full paraphenalia of mean field electrodynamics, including a turbulent magnetic diffusivity $\eta_{T}$ and perhaps an $\alpha$-effect, be invoked?"

On the one hand, the answer is clearly "No". Although the macroscale magnetic Reynolds number $R m$ is necessarily large, it is apparently not very large. If $R m=100$ and $\ell / \mathcal{L}=1 / 3000$ (see above) and, if the characteristic turbulent velocity $\mathcal{V}^{\prime}$ is of the same order as the characteristic macroscale velocity $\mathcal{V}$, the microscale Reynolds number, $R m^{\prime} \equiv \mathcal{V}^{\prime} \ell / \nu_{M}$, is only about 0.03. Moreover, a drastic increase in $\eta$ from the currently favored value of $\eta_{M} \approx 2 \mathrm{~m}^{2} \mathrm{~s}^{-1}$ to $\eta_{\text {total }}=\eta_{T}+\eta_{M}$ where $\eta_{\text {total }} \gg \eta_{M}$ would result in a corresponding reduction in the time-scale of the macroscale field for which, in the case of the Earth, there is no observational evidence. The study of paleomagnetism has shown that a polarity reversal of the Earth's field is accomplished in a time of the order of 5,000 yr or greater, which is comparable with the free-decay time, $\tau_{\eta} \equiv \mathcal{L}^{2} / \pi^{2} \eta_{M} \approx 20,000 \mathrm{yr}$, of the dipole field in the absence of turbulence. This situation may be contrasted with that for the Sun, for which $\eta_{T}$ exceeds $\eta_{M}$ by a very large factor, and for which the time-scale of magnetic activity is enormously less than $\tau_{\eta}$.

On the other hand, all numerical simulations that successfully imitate the Earth by exhibiting polarity reversals have been driven strongly enough to be irregular; see for example Glatzmaier and Roberts (1995a,b, 1996a,b) and Sarson and Jones (1999). The fields fluctuate in time, and there is a suspicion that a polarity reversal is merely another fluctuation that happens to be rather larger than the others. Such irregularities in $\overline{\mathbf{B}}$ suggest that fluctuations in $\mathbf{B}^{\prime}$ may be significant also, and especially so because the associated electric current density $\mathbf{J}^{\prime}=\nabla \times \mathbf{B}^{\prime} / \mu_{0} \sim \mathcal{B}^{\prime} / \mu_{0} \ell$ (where $\mu_{0}$ is the magnetic 
permeability) is enhanced relative to $\overline{\mathbf{J}}=\nabla \times \overline{\mathbf{B}} / \mu_{0} \sim \overline{\mathcal{B}} / \mu_{0} \mathcal{L}$ by the factor $\mathcal{L} / \ell$, so that $\mathcal{J}^{\prime} / \overline{\mathcal{J}} \sim(\mathcal{L} / \ell) \mathcal{B}^{\prime} / \overline{\mathcal{B}} \sim 3000 \mathcal{B}^{\prime} / \overline{\mathcal{B}}$. This means that the Joule losses per unit volume, $q^{J}=$ $\mu_{0} \eta_{M} \mathbf{J}^{2}$, of the electric currents $\mathbf{J}$ may have a significant microscale part $q^{J^{\prime}}=\mu_{0} \eta_{M} \overline{\left(\mathbf{J}^{\prime}\right)^{2}}$ in addition to the obvious macroscale contribution $q^{\bar{J}}=\mu_{0} \eta_{M} \overline{\mathbf{J}}^{2}$. This is confirmed in $\S 2$ below, where we show that the smaller scale components of the magnetic field make a bigger contribution to the Joule losses than the lowest order components such as the dipole field. We are able to glean information on how rapidly the field components fall off with increasing wavenumber, and hence to estimate the total Joule dissipation, albeit rather approximately. Our arguments suggest that, although the turbulent magnetic diffusivity exceeds the molecular magnetic diffusivity, it does not do so by a large factor.

Our estimate of $q^{J}$ has a strong bearing on the thermodynamic arguments to be given in $\S \S 4$ and 5 . These are based on a model of the core which is developed in $\S 2$. In $\S 6$ we present a case, based purely on considerations of core thermodynamics for the existence of significant radioactivity in the core.

\section{OHMIC DISSIPATION}

\subsection{Order of magnitude estimates}

Glatzmaier and Roberts (1996a) estimated that the contribution, $\mathcal{Q}^{\overline{\mathcal{J}}}$, to the total ohmic dissipation $\mathcal{Q}^{J}$ made by the large scale fields in their simulation was on average about

$$
\mathcal{Q}^{\overline{\mathcal{J}}}=0.3 \mathrm{TW}
$$

A crude argument suggests that this is much less than $\mathcal{Q}^{J^{\prime}}$. Fluctuations of magnetic field $\mathbf{B}^{\prime}$ on a short length-scale $\ell$ produced by velocity fluctuations $\mathbf{V}^{\prime}$ acting on a large scale mean field $\overline{\mathbf{B}}$ satisfy

$$
\nabla \times\left(\mathbf{V}^{\prime} \times \overline{\mathbf{B}}\right) \sim \eta_{M} \nabla^{2} \mathbf{B}^{\prime}, \quad \text { or } \quad B^{\prime} \sim \frac{V^{\prime} \ell}{\eta_{M}} \bar{B}
$$

The large-scale and small-scale dissipations are then

$$
\mathcal{Q}^{\overline{\mathcal{J}}} \sim \frac{\eta_{M} \bar{B}^{2}}{\mu_{0} \mathcal{L}^{2}} \mathcal{V}_{\mathrm{FOC}}, \quad \mathcal{Q}^{J^{\prime}} \sim \frac{\eta_{M} B^{\prime 2}}{\mu_{0} \ell^{2}} \mathcal{V}_{\mathrm{FOC}}
$$

where $\mathcal{V}_{\text {FOC }}$ is the volume of the fluid outer core (FOC). If we further assume that the fluctuating velocities $\mathbf{V}^{\prime}$ are of the same order as the large scale velocity $\mathcal{V}$, we find that

$$
\frac{\mathcal{Q}^{J^{\prime}}}{\mathcal{Q}^{\overline{\mathcal{J}}}} \sim \frac{B^{\prime 2} \mathcal{L}^{2}}{\bar{B}^{2} \ell^{2}} \sim\left(\frac{\mathcal{V} \mathcal{L}}{\eta_{M}}\right)^{2}=R_{m}^{2}
$$

Since $R_{m}$ is at least 100 in the core, this suggests $\mathcal{Q}^{J^{\prime}} \sim 10^{4} \mathcal{Q}^{\overline{\mathcal{J}}}$. Equation (2.1) would then imply that $\mathcal{Q}^{J} \approx \mathcal{Q}^{J^{\prime}} \sim 3000 \mathrm{TW}$, which is clearly far too large.

Some reduction in $\mathcal{Q}^{J^{\prime}}$ could be obtained if turbulence fills only part of the FOC, or is strongly intermittent. However, the estimate (2.3) can also be questioned. It is essentially kinematic in origin; Lorentz forces may reduce the cross-field velocity, hence 
reducing $\mathbf{V}^{\prime} \times \overline{\mathbf{B}}$. Some evidence for this is present in plane-layer dynamo calculations (Jones and Roberts 2000). One may also object that (2.1) comes from a simulation that includes 'intermediate' length scales (down to around $100 \mathrm{~km}$ ), and so it already includes some of the smaller scale dissipation. In other words, a substantial part of $\mathcal{Q}^{\overline{\mathcal{J}}}$ should be re-assigned to $\mathcal{Q}^{J^{\prime}}$.

There is a common perception that the geodynamo is of strong field type, meaning that the Coriolis and Lorentz forces associated with the large-scale fields are of the same order of magnitude, so that $\bar{J}$ is of order $2 \Omega \rho \mathcal{V} / \bar{B}$. The simulations that led to (2.1) gave typical field strengths of order $10^{-2} \mathrm{~T}$, and, taking $\rho=10^{4} \mathrm{~kg} \mathrm{~m}^{-3}$ and $\mathcal{V}=10^{-4} \mathrm{~m} \mathrm{~s}^{-1}$, we find that $\bar{J}$ is of order $1.4 \times 10^{-2} \mathrm{~A} \mathrm{~m}^{-2}$. Taking $\eta_{M}=2 \mathrm{~m}^{2} \mathrm{~s}^{-1}$, we obtain $\mathcal{Q}^{\overline{\mathcal{J}}}=0.08$ TW. This suggests that $75 \%$ of the $0.3 \mathrm{TW}$ given in (2.1) belongs in $\mathcal{Q}^{J^{\prime}}$. Glatzmaier and Roberts (1996a) obtained (2.1) from the scales that they resolved in their computation, which included all spherical harmonics up to degree 21, corresponding to length scales of about $100 \mathrm{~km}$. They described the remainder as 'turbulent dissipation' and assigned it to their unresolved scale; they assessed it at about 0.1 TW. Their simulation, in common with most of the other simulations of strongly-driven dynamos, employed hyperdiffusion, which artificially damps out the shorter length scales, and decreases $\mathcal{Q}^{J^{\prime}}$ artificially relative to $\mathcal{Q}^{\overline{\mathcal{J}}}$. This substantially increases the rate at which the magnetic energy spectrum decreases with increasing harmonic number. (Compare the spectrum from their simulation with that of the highly resolved model reported in Roberts and Glatzmaier (2000a), which does not employ hyperdiffusion.) It is therefore likely that the contribution made to $\mathcal{Q}^{J}$ by their unresolved scales was unrepresentatively small and that, if hyperdiffusion had been eliminated, $\mathcal{Q}^{J}$ would have been much larger than $0.4 \mathrm{TW}$.

Although scale separation is convenient and often used, it is an oversimplification. In reality 'intermediate scales' are significant, as we shall demonstrate in $\S 2.2$. This leads to an ambiguity in the interpretation of results like (2.1) in two-scale language.

\subsection{Large-scale dissipation in the core}

We first consider the minimum dissipation in the core associated with the observed components of the geomagnetic field. Only the poloidal part of the field is visible at the surface, so no toroidal field is included in this minimum dissipation calculation. Gubbins (1977) showed that the minimum dissipation associated with the geomagnetic field is

$$
\mathcal{Q}^{J}=\sum_{n=1}^{\infty} q_{n}, \quad q_{n}=\frac{\eta_{M} r_{\mathrm{CMB}}}{\mu_{0}} \frac{(2 n+1)(2 n+3)}{n} R_{n}
$$

where $q_{n}$ is the dissipation from the spherical harmonics of order $n$, and

$$
R_{n}=\left(\frac{r_{\mathrm{E}}}{r_{\mathrm{CMB}}}\right)^{2 n+4}(n+1) \sum_{m=0}^{n}\left[\left(g_{n}^{m}\right)^{2}+\left(h_{n}^{m}\right)^{2}\right]
$$

is the Mauersberger-Lowes spectrum extrapolated to the core surface (see e.g., Langel 1987), $r_{\mathrm{E}}$ and $r_{\mathrm{CMB}}$ being the radii of the Earth and of the core-mantle boundary (CMB); $g_{n}^{m}$ and $h_{n}^{m}$ are the usual Gauss coefficients that multiply Schmidt-normalized associated Legendre functions in the expansion of the magnetic potential at the Earth's surface (see 
e.g., Backus et al. 1996). Using MAGSAT values of the Gauss coefficients and our standard estimate of $\eta=2 \mathrm{~m}^{2} \mathrm{~s}^{-1}$, we find that

$$
q_{1}=5.82 \mathrm{MW} \text { and } q_{2}=0.75 \mathrm{MW} .
$$

However, as $n$ increases past 2 , the dissipation starts to increase with $n$, reaching a maximum at $q_{8}$ of 1.5 MW. This strongly suggests that the 'intermediate' scales contribute significantly to the total dissipation. Observations indicate that the Mauersberger-Lowes spectrum $R_{n}$ at the CMB is well-approximated for $n \geq 3$ by

$$
R_{n}=1.51 \times 10^{-8} \exp (-0.1 n) \mathrm{T}^{2}
$$

and using (2.6)-(2.8) we can derive an estimate of the minimum total dissipation associated with the observed field:

$$
\mathcal{Q}_{\text {min }}^{J}=\sum_{n=1}^{\infty} q_{n}=q_{1}+q_{2}+\frac{\eta_{M} r_{\mathrm{CMB}}}{\mu_{0}} \sum_{n=3}^{\infty} \frac{(2 n+1)(2 n+3)}{n} R_{n},
$$

which by (2.9) gives $\mathcal{Q}_{\text {min }}^{J} \approx 44 \mathrm{MW}$. This agrees well with the estimate of Roberts and Glatzmaier $(2000 \mathrm{~b}): \mathcal{Q}_{\min }^{J} \approx 43 \mathrm{MW}$. It should be noted that a significant part of $\mathcal{Q}_{\text {min }}^{J}$ (about $25 \mathrm{MW}$ ) is made by modes with $n>12$, which is beyond the testable range of (2.9), because the core field signal is then swamped by crustal field contributions.

The actual magnetic field has to satisfy the MHD equations, a constraint not imposed in the derivation of (2.6). A somewhat more realistic estimate can be obtained from the dissipation associated with poloidal and toroidal decay modes (see e.g., Moffatt 1978). The ohmic dissipation associated with the poloidal modes is bounded below by the dissipation of the decay modes that have the same power spectra at the CMB. From this consideration, we see that $q_{n}$ cannot be smaller than

$$
q_{n}=\frac{2 \pi \eta_{M} r_{\mathrm{CMB}}}{\mu_{0}} \frac{\alpha_{n-1}^{4}}{n(2 n+1)} R_{n},
$$

where $\alpha_{n}$ is the smallest positive zero of the spherical Bessel function, $j_{n}(\alpha)$. This gives as lower limits,

$$
q_{1}=79.5 \mathrm{MW} \text { and } q_{2}=11.0 \mathrm{MW} .
$$

We can again use the Mauersberger-Lowes spectrum (2.9) to estimate the total dissipation. As with (2.6), the dissipation increases with $n$ when $n \geq 3$, reaching a maximum of 36.2 MW, this time of $n=14$. We can use the first three terms of the asymptotic formula for the first zero of the Bessel function (e.g., Abramowitz and Stegun 1970):

$$
\alpha_{n} \sim\left(n+\frac{1}{2}\right)+1.85576\left(n+\frac{1}{2}\right)^{1 / 3}+1.03315\left(n+\frac{1}{2}\right)^{-1 / 3}
$$

to obtain an alternative, and more stringent, lower bound

$$
\mathcal{Q}_{\text {min }}^{J}=\sum_{n=1}^{\infty} q_{n} \approx q_{1}+q_{2}+\sum_{n=3}^{\infty} q_{n} \approx 1.32 \mathrm{GW}
$$


where now (2.11)-(2.13) are used to evaluate (2.14). Note that the higher harmonics make an even greater contribution to the dissipation than was the case for the 'minimizing' field. This is because, as (2.13) shows, $\alpha_{n}$ is linear with $n$ in the large $n$ limit, so the dissipation is asymptotically proportional to $n^{2} \exp (-0.1 n)$ for the decay modes, but only proportional to $n \exp (-0.1 n)$ for the 'minimizing' field. Of course, the caveats mentioned above about the uncertainty of the Mauersberger-Lowes spectrum at $n=14$ apply with even more force in this case. Nevertheless, it is interesting that this spectrum suggests that the higher $n$ modes dissipate over 16 times the energy than the $n=1$ (dipole) mode.

In view of the fact that the observed spectrum (2.9) is based only on observations of modes with $n<12$, it is of interest to compare (2.9) with spectra derived from geodynamo simulations. A recent simulation (Glatzmaier \& Roberts 2000a), using a very large number of modes but run for a comparatively short time, gave a spectrum of the form (2.9) but with an exponent -0.055 instead of -0.1 . Although the run was short (only about 1500 yrs), this is sufficient to establish the power spectrum, and harmonics up to $n=239$ were included. The value of viscosity used naturally had to exceed that of the Earth by a considerable factor, but nevertheless it is small enough for the great bulk of the dissipation to be ohmic rather than viscous. We therefore consider how (2.14) is affected if the spectrum

$$
R_{n}=1.51 \times 10^{-8} \exp (-0.055 n) \mathrm{T}^{2}
$$

is used in place of (2.9). We have left the pre-factor unchanged, as this does not greatly affect the lower order modes, which are the most confidently known. The effect is to increase the dissipation in (2.14) by over a factor 4 to $5.77 \mathrm{GW}$, with the peak now occurring at $n=29$ rather than $n=14$.

We can also derive some estimates for the losses from toroidal modes, though here we have to rely on numerical simulations to estimate the strength of the toroidal fields. The dissipation can be computed directly from these simulations, but as this is not always done it is of interest to relate the dissipation directly to the average field strengths in the simulations, which are usually given.

The toroidal decay modes can be written

$$
\mathbf{B}=\boldsymbol{\nabla} \times\left[T_{n m} j_{n}(k r) P_{n}^{m}(\cos \theta) \exp (i m \phi) \mathbf{r}\right],
$$

where $k=\alpha / r_{\mathrm{CMB}}$. The root mean square magnetic field associated with this mode is

$$
\bar{B}^{2}=\frac{1}{\mathcal{V}_{\text {core }}} \int_{\text {core }} \mathbf{B}^{2} d^{3} x=\frac{3 n(n+1)}{2(2 n+1)}\left[j_{n}^{\prime}(\alpha)\right]^{2}\left|T_{n m}\right|^{2}
$$

where $\mathcal{V}_{\text {core }}$ is the volume of the core. The right-hand side of $(2.17)$ is proportional to the ohmic dissipation produced by the mode, which is

$$
q_{n}^{m}=\frac{\eta_{M} r_{\mathrm{CMB}}}{\mu_{0}} \frac{2 n(n+1)}{2 n+1}\left[\alpha j_{n}^{\prime}(\alpha)\right]^{2}\left|T_{n m}\right|^{2}=\frac{4 \pi r_{\mathrm{CMB}} \eta_{M} \alpha^{2}}{3 \mu_{0}} \bar{B}^{2} .
$$

The lowest order toroidal mode with dipole symmetry $\left(B_{\phi}\right.$ antisymmetric about the equator) is $n=2, m=0$ for which the smallest non-trivial $\alpha$ is 5.7635. With our standard 
estimates this gives $q_{2}=7.7 \times 10^{14} \bar{B}^{2} \mathrm{~W}$. The Glatzmaier-Roberts simulations had a toroidal field of order 100 gauss, which is $0.01 \mathrm{~T}$, giving a dissipation $q_{2} \sim 0.077 \mathrm{TW}$, about one quarter of the dissipation (2.1). If the spectrum for the toroidal modes follows the same power law (2.15) as the poloidal modes (with a different prefactor) the total power consumption will be almost 20 times greater, i.e., about 1.5 TW.

The dissipation coming from the toroidal modes seems to be much larger than that from the poloidal modes (2.14). However, this is because our estimate for the poloidal field comes from the observed Gauss coefficients, which correspond to a field strength of only $0.05-0.1 \mathrm{mT}$. All simulations suggest that the poloidal field is considerably stronger than this, with most of the poloidal field forming closed field lines that do not escape from the FOC, and hence do not contribute to the Gauss coefficients. We estimate that the toroidal and poloidal field together give a total ohmic power loss in the range

$$
1 \mathrm{TW}<\mathcal{Q}^{J}<2 \mathrm{TW}
$$

In most of what follows, (2.19) is more significant than (2.1); the latter is of interest only in $\S 5$.

\section{THE ADIABATIC STATE}

\subsection{Seismically determined variables}

In this section we describe how the estimates to be presented in $\S 4$ were obtained. We suppose that the fluid alloy is, to the first approximation, in a well-mixed hydrostatic state under its own self-produced gravitational field $\mathbf{g}_{a}$ :

$$
\nabla P_{a}=\rho_{a} \mathbf{g}_{a}, \quad \nabla S_{a}=\mathbf{0}, \quad \nabla \xi_{a}=\mathbf{0},
$$

where $P$ is pressure and $\rho$ is density; the suffix ${ }_{a}$ refers to the fact that this is an adiabatic state, by (3.2). Other (intensive) variables are not homogenized by the mixing. In the case of the temperature $T$ for example,

$$
T_{a}^{-1} \nabla T_{a}=\gamma \mathbf{g}_{a} / u_{P}^{2}, \quad \text { where } \quad \gamma=\alpha u_{P}^{2} / c_{p}
$$

is the Grüneisen parameter, $u_{P}$ is the speed of sound, $\alpha$ is the coefficient of thermal expansion, and $c_{p}$ is the specific heat at constant pressure.

It is possible to use (3.1)-(3.3) to construct models of the Earth based on seismic data. Usually the centrifugal force is ignored, so that $\mathbf{g}_{a}$ is radial and all variables depend spatially only on the distance $r$ from the geocentre. One such model is the preliminary reference Earth model (PREM) of Dziewonski and Anderson (1981); another is the model ak135 of Kennett et al. (1995). Both papers provide the seismic velocities and the implied $\rho$ as functions of $r$, and in the case of PREM these are given as useful polynomial expressions. In their 1996 and subsequent papers, Glatzmaier and Roberts (1996a) reported the results of dynamo simulations based on PREM and on the underlying theoretical formulation of Braginsky and Roberts (1995) of thermodynamically self-consistent magnetoconvection.

Our procedure was the following: We integrated (to second order accuracy) the differential equations

$$
\frac{d \rho_{a}}{d r}=-\frac{\rho_{a} g_{a}}{\Phi}, \quad \frac{d}{d r}\left(r^{2} g_{a}\right)=4 \pi G r^{2} \rho_{a}
$$


where the seismic parameter $\Phi=u_{P}^{2}-\frac{4}{3} u_{S}^{2}$ was derived from the values of the seismic velocities $u_{P}$ and $u_{S}$ tabulated in ak135; the same density discontinuities as ak135 were assumed, namely $209 \mathrm{~kg} \mathrm{~m}^{-3}$ at $410 \mathrm{~km}$ depth, $306 \mathrm{~kg} \mathrm{~m}^{-3}$ at $660 \mathrm{~km}$ depth, $4364 \mathrm{~kg}$ $\mathrm{m}^{-3}$ at the $\mathrm{CMB}$ and $\Delta \rho \equiv \rho_{\mathrm{SIC}}-\rho_{\mathrm{FOC}}=565 \mathrm{~kg} \mathrm{~m}^{-3}$ at the inner core boundary (ICB), 'SIC' being an abbreviation for 'solid inner core'. This $\Delta \rho$ agrees well with that of Shearer and Masters (1991) who derived $550 \pm 50 \mathrm{~kg} \mathrm{~m}^{-3}$. (The discontinuities of $400 \mathrm{~kg} \mathrm{~m}^{-3}$ at $35 \mathrm{~km}$ depth and $200 \mathrm{~kg} \mathrm{~m}^{-3}$ at $20 \mathrm{~km}$ depth were also included.) Equation (3.5) is the scalar form of (3.1) with $\partial P / \partial \rho$ replaced by $K / \rho$, where $K=\rho \Phi$ is the incompressibility. Equation (3.6) is the Poisson equation determining $g_{a}$; here $G$ is the gravitational constant. An arbitrary initial value for the central density $\rho_{c}$ was assumed, and the equations were iterated until the correct total mass for the Earth was obtained. The values derived for $\rho$ did not differ substantially from those tabulated in ak135. For example, we found the following: $\rho_{c}=13054 \mathrm{~kg} \mathrm{~m}^{-3} ; \rho_{\mathrm{SIC}}=12728 \mathrm{~kg} \mathrm{~m}^{-3}$ and $\rho_{\mathrm{FOC}}=12164 \mathrm{~kg} \mathrm{~m}^{-3}$ at the ICB; $\rho_{\mathrm{FOC}}=9893 \mathrm{~kg} \mathrm{~m}^{-3}$ and $\rho_{\text {mantle }}=5529 \mathrm{~kg} \mathrm{~m}^{-3}$ at the CMB. The corresponding ak135 values are 12892, 12704, 12139, 9915 and $5551 \mathrm{~kg} \mathrm{~m}^{-3}$, respectively. We also derived the following results, which are close to the corresponding PREM results, the latter being given in square brackets for comparison. [In the case of PREM, we did not integrate (3.5) and (3.6), but used the polynomial expressions for $\rho_{a}$ given by the authors.] The central pressure is $P_{c}=363$ [361] GPa, $P_{\mathrm{ICB}}=328$ [329] GPa, $P_{\mathrm{CMB}}=135$ [136] GPa, the incompressibility $K_{\mathrm{ICB}}=1.29[1.30] \mathrm{GPa}, g_{\mathrm{ICB}}=4.38[4.40] \mathrm{m} \mathrm{s}^{-2}, g_{\mathrm{CMB}}=10.68[10.68]$ $\mathrm{m} \mathrm{s}^{-2}$ and at the Earth's surface $g=9.81 \mathrm{~m} \mathrm{~s}^{-2}$; these values are close to those generally accepted. We found that the gravitational energy of the entire Earth is $2.495[2.487] \times 10^{33}$ $\mathrm{J}$, and the gravitational potential $U_{a}$ is $1.116[1.117] \times 10^{8} \mathrm{~m}^{2} \mathrm{~s}^{-2}$ at the geocenter. An integral of interest below is

$$
\mathcal{E}_{\mathrm{FOC}}=\int_{\mathrm{FOC}}\left(U_{\mathrm{ICB}}-U_{a}\right) \rho_{a} d^{3} x .
$$

We found this to be $1.818[1.817] \times 10^{31} \mathrm{~J}$.

\subsection{Adiabatic temperature distribution}

We introduced an empirical law for $\gamma$, namely that of Merkel et al. (2000):

$$
\gamma=\gamma_{0}\left(\frac{\rho_{0}}{\rho}\right)^{q}
$$

where $\gamma_{0}=1.68$ is the Grüneisen parameter for decompressed iron $\left(\rho_{0}=7070 \mathrm{~kg} \mathrm{~m}^{-3}\right)$ and $q=0.7$. This led to $\gamma_{\mathrm{ICB}}=1.15$ and $\gamma_{\mathrm{CMB}}=1.33$. Laio et al. (2000) give the melting point of pure iron at the ICB pressure as $5400^{\circ} \mathrm{K}$. The depression, $\Delta T_{m}$, of the melting point through alloying is very uncertain. Williams et al. (1987) estimate that $\Delta T_{m}=1000^{\circ} \mathrm{K}$, while Jeanloz (1990) states that $\Delta T_{m}=1000 \pm 1000^{\circ} \mathrm{K}$. Boehler (1996) argues that $\Delta T_{m}$ decreases with increasing pressure and is probably small, at most of the order of a few $100^{\circ} \mathrm{K}$, at the ICB. Braginsky and Roberts (1996) worked with $\Delta T_{m}=700^{\circ} \mathrm{K}$. We took $\Delta T_{m}=300^{\circ} \mathrm{K}$, giving an anchor point $T_{\mathrm{ICB}}=5400-\Delta T_{M}=5100^{\circ} \mathrm{K}$ for the integration of (3.4). To second order accuracy, we found that

$$
T_{\mathrm{CMB}}=3949[3954]^{\circ} \mathrm{K}, \quad \bar{T}=4488[4486]^{\circ} \mathrm{K},
$$


where $\bar{T}$ is the mass-weighted average of $T$ in the FOC; the volume averaged $T$ is 4470 $[4468]^{\circ} \mathrm{K}$. The adiabatic gradients at the FOC boundaries were $0.24[0.25]^{\circ} \mathrm{K} \mathrm{km}^{-1}$ and 0.88 $[0.85]^{\circ} \mathrm{K} \mathrm{km}^{-1}$. In $\S 4.2$ we will encounter $\widetilde{T}$, which is the reciprocal of the mass-weighted average of $1 / T_{a}$. For ak135 this is $4465^{\circ} \mathrm{K}$, which differs little from $\bar{T}$.

\subsection{Thermal conductivity}

From our assumed value $\eta_{M}=2 \mathrm{~m}^{2} \mathrm{~s}^{-1}$ of the magnetic diffusivity and the WiedemannFranz law (with a value of the Lorentz constant of $0.0194 \mathrm{~W} \mathrm{~m} \mathrm{~s}^{-2}{ }^{\circ} \mathrm{K}^{-2}$ ) we obtained a thermal conductivity $K_{T}$ at the $\mathrm{CMB}$ of $38 \mathrm{~W} \mathrm{~m}^{-1}{ }^{\circ} \mathrm{K}^{-1}$. Stacey and Anderson (2001) supplement this electronic part with a lattice contribution, leading them to $46 \mathrm{~W} \mathrm{~m} \mathrm{~m}^{-1}$ ${ }^{\circ} \mathrm{K}^{-1}$. We dealt with this point by multiplying our $K_{T}$ everywhere by the appropriate constant factor, giving for example $K_{T}=57 \mathrm{~W} \mathrm{~m}^{-1}{ }^{\circ} \mathrm{K}^{-1}$ at the ICB. Having derived in this way $K_{T}$ as a function of $r$, we could determine the adiabatic heat flux, which we found to be $0.0138[0.0141] \mathrm{W} \mathrm{m}^{-2}$ at the ICB and $0.0386[0.0373] \mathrm{W} \mathrm{m}^{-2}$ at the CMB. The corresponding net adiabatic heat flows are

$$
\mathcal{H}_{\mathrm{ICB}}=0.26[0.26] \mathrm{TW}, \quad \mathcal{H}_{\mathrm{CMB}}=5.87[5.68] \mathrm{TW} .
$$

The expenditure of entropy by the adiabatic gradient in the FOC is

$$
\Sigma=\int_{\mathrm{FOC}} K_{M}\left(\nabla T_{a} / T_{a}\right)^{2} d V \approx 172[167] \mathrm{MW}^{\mathrm{o}} \mathrm{K}^{-1}
$$

This estimate omits the contributions to $\Sigma$ from boundary layers, which are assumed to be negligible.

\subsection{Specific and latent heats; contraction on solidification}

We derived estimates of other thermal properties in the FOC, as functions of $r$, by the following process. To compute $c_{p}$ and $\alpha$, we adopted the approach of Braginsky and Roberts (1995), who took $c_{v}$, the specific heat at constant volume, to be $670 A^{\mathrm{Fe}} / \bar{A} \mathrm{~J} \mathrm{~kg}-1$ ${ }^{\circ} \mathrm{K}^{-1}$, where $A^{\mathrm{Fe}}=55.85$ is the atomic weight of iron and $\bar{A}=48.1$ is the mean atomic weight of the core (see e.g., Stacey, 1992). From this they derived $c_{p}$ from equation (E7) of Braginsky and Roberts (1995):

$$
c_{p}=\frac{c_{v}}{1-\gamma^{2} c_{v} T / u_{P}^{2}} .
$$

They then obtained $\alpha=\gamma c_{p} / u_{P}^{2}$.

We found that, at the ICB, $c_{p}=819[820] \mathrm{J} \mathrm{kg}^{-1}{ }^{\circ} \mathrm{K}^{-1}$ and $\alpha=0.89[0.90] \times 10^{-5 \circ} \mathrm{K}^{-1}$; at the $\mathrm{CMB}, c_{p}=850[848] \mathrm{J} \mathrm{kg}^{-1} \mathrm{o}^{-1}$ and $\alpha=1.77[1.70] \times 10^{-5 \circ} \mathrm{K}^{-1}$. Having found $c_{p}$, we can transform the thermal conductivity $K_{T}$ into a thermal diffusivity $\kappa_{M}=K_{T} / \rho c_{p}$, and we find that $\left(\kappa_{M}\right)_{\mathrm{ICB}}=5.7 \times 10^{-6} \mathrm{~m}^{2} \mathrm{~s}^{-1}$ and $\left(\kappa_{M}\right)_{\mathrm{CMB}}=5.4 \times 10^{-6} \mathrm{~m}^{2} \mathrm{~s}^{-1}$.

We follow Anderson and Duba (1997) by supposing that the change in specific volume on freezing is $2.0 \times 10^{-6} \mathrm{~m}^{3} \mathrm{~kg}^{-1}$, corresponding to a contribution of $\Delta^{s} \rho=310 \mathrm{~kg} \mathrm{~m}^{-3}$ to $\Delta \rho$, the remainder, $\Delta^{\xi} \rho=255 \mathrm{~kg} \mathrm{~m}^{-3}$, being due to the rejection of light constituent on freezing core mix. Braginsky and Roberts (1995) divided the PREM value of $\Delta \rho=600$ $\mathrm{kg} \mathrm{m}^{-3}$ differently, taking $\Delta^{s} \rho=100 \mathrm{~kg} \mathrm{~m}^{-3}$ and $\Delta^{\xi} \rho=500 \mathrm{~kg} \mathrm{~m}^{-3}$. This is the primary reason why our estimates below differ from theirs. 
It may be worth pointing out that the smaller value of $\Delta^{\xi} \rho$ assumed by Anderson and Duba (1997) as compared with Braginsky and Roberts (1995) diminishes the potency of compositional buoyancy. Thermal buoyancy becomes more significant and plausibly the character of the geodynamo does not undergo as marked a change as the SIC comes into existence. If a similar situation arises in the core of Venus, one may speculate that the possible absence of an inner core in that planet may not explain why it does not create a magnetic field. (For an alternative point of view, see Stevenson et al. 1983.)

We used the expression for the latent heat of crystallization, $L$, given by Anderson and Duba (1997):

$$
L=\left[\frac{K}{2\left(\gamma-\frac{1}{3}\right)\left(1+\gamma^{2} \alpha T\right)}\right]_{\mathrm{ICB}} \Delta^{s} V .
$$

This gives $L=1.56 \times 10^{6} \mathrm{~J} \mathrm{~kg}^{-1}$, instead of the value of $10^{6} \mathrm{~J} \mathrm{~kg}^{-1}$ of Braginsky and Roberts (1995), which they drew from Gubbins et al. (1979). Labrosse et al. $(1997,2001)$ took $L=0.625 \times 10^{6} \mathrm{~J} \mathrm{~kg}^{-1}$, which they derived from Poirier and Shankland (1993) using $\Delta^{s} V=0.93 \mathrm{~m}^{3} \mathrm{~kg}^{-1}$. Laio et al. (2000) obtained $L=0.7 \times 10^{6} \mathrm{~J} \mathrm{~kg}^{-1}$.

\subsection{Light constituent}

Braginsky and Roberts (1995) argued for a value of about 0.16 for the mass fraction $\xi_{a}$ of light constituent in the well-mixed adiabatic state but later research (e.g., Anderson and Duba 1997) suggests that $10 \%$ is a better estimate; Loper (1991) took $9 \%$. In what follows, we take $\xi_{a}=0.095$. Braginsky and Roberts (1995) related $\xi_{a}$ to $\Delta \xi$, where $\Delta \xi \equiv \xi_{\mathrm{FOC}}-\xi_{\mathrm{ICB}}$ is the discontinuity in $\xi$ at the ICB, by a 'rejection factor', $r_{\mathrm{FS}}=\Delta \xi / \xi_{a}$ and showed that, to a good approximation,

$$
r_{\mathrm{FS}} \equiv \frac{\Delta \xi}{\xi_{a}}=\frac{\Delta^{\xi} \rho / \rho_{\mathrm{SIC}}}{1-\rho / \rho_{H}}
$$

where $\rho / \rho_{H}$ is the ratio of $\rho$ to the density that pure iron would have under the same conditions. They presented an argument that led to the estimate $\rho_{H} / \rho=1.1052$, which we adopt for the SIC. This leads from $\xi_{a}=0.095$ to $\Delta \xi=0.020$. Note that this value of $\Delta \xi$ is significantly less than the estimate of 0.06 given in Braginsky and Roberts (1995). The change is due partly to the revised partition of $\Delta \rho$ and partly due to the reduced estimate of $\xi_{a}$, both changes decreasing the estimated $\Delta \xi$.

A further significant parameter is the compositional expansion coefficient, $\alpha^{\xi}$, which at the bottom of the FOC is

$$
\alpha^{\xi} \equiv \frac{1}{\rho}\left(\frac{\partial \rho}{\partial \xi}\right)_{P, S}=\frac{\Delta^{\xi} \rho}{\rho_{\mathrm{SIC}} \Delta \xi}
$$

here, as in (3.14) and in (3.18) below, $\rho_{\text {SIC }}$ refers to the top of the SIC. We suppose that the value $\alpha^{\xi}=1.0$ obtained from (3.15) holds throughout the FOC; this may be compared with $\alpha^{\xi}=0.6$, as used by Braginsky and Roberts (1995).

A quantity that is significant when discussing the advance of the ICB through freezing is

$$
\Delta_{2}=\Delta_{m a}+\Delta_{m \xi},
$$


where

$$
\begin{aligned}
\Delta_{m a} & =\left(\frac{\gamma g r}{u_{P}^{2}}\right)_{\mathrm{ICB}}\left(\frac{\partial T_{m} / \partial P}{\partial T_{a} / \partial P}-1\right), \\
\Delta_{m \xi} & =-\frac{3 \mathcal{V}_{\mathrm{SIC}} \rho_{\mathrm{SIC}} \Delta \xi}{\mathcal{M}_{\mathrm{FOC}} T}\left[\frac{h^{\xi}}{c_{p}}+\frac{\partial T_{m}}{\partial \xi}\right]
\end{aligned}
$$

Here $\mathcal{V}_{\mathrm{SIC}} \approx 1.765 \times 10^{20} \mathrm{~m}^{3}$ is the volume of the SIC and $\mathcal{M}_{\mathrm{FOC}} \approx 1.8412 \times 10^{24} \mathrm{~kg}$ is the mass of the FOC. We took the heat of reaction, $h^{\xi}$, to be $-1.6 \times 10^{7} \mathrm{~J} \mathrm{~kg}^{-1}$, a value quoted by Gubbins (1977) for FeS; Braginsky and Roberts (1995) took $h^{\xi}=-5 \times 10^{6} \mathrm{~J}$ $\mathrm{kg}^{-1}$ for this rather uncertain parameter. We use the crude estimate $\partial T_{m} / \partial \xi=\Delta T_{m} / \Delta \xi$. Recalling that we are assuming $\Delta T_{m}=300^{\circ} \mathrm{K}$, we find that $\Delta_{m \xi}=0.014$. Estimating the melting point gradient $\partial T_{m} / \partial P$ by using Lindemann's law, which Braginsky and Roberts (1995) showed reduces approximately to

$$
\frac{\partial T_{m} / \partial P}{\partial T_{a} / \partial P}-1=1-\frac{2}{3 \gamma}
$$

we obtain $\Delta_{m a}=0.024$, giving $\Delta_{2}=0.038$ [0.038] in total. Braginsky and Roberts obtained $\Delta_{m a}=0.03, \Delta_{m \xi}=0.02$, and $\Delta_{2}=0.05$ in total.

\section{ENERGY AND ENTROPY}

\subsection{Energy balance}

The energy balance of the FOC is expressed by

$$
\mathcal{Q}_{\mathrm{CMB}}=\mathcal{Q}_{\mathrm{ICB}}+\mathcal{Q}^{L}+\mathcal{Q}^{S}+\mathcal{Q}^{G}+\mathcal{Q}^{R}
$$

where $\mathcal{Q}_{\mathrm{ICB}}$ and $\mathcal{Q}_{\mathrm{CMB}}$ are the heat flows in and out of the boundaries, and $\mathcal{Q}^{L}$ is the latent heat released at the SIC into the FOC. The remaining terms are volumetric sources within the FOC: $\mathcal{Q}^{R}$ is the rate of supply of the radioactive sources; $\mathcal{Q}^{S}$ is rate of diminution of internal energy through cooling; and $\mathcal{Q}^{G}$ is the gravitational energy released through the emission of light constituent during freezing at the ICB, this constituent being mixed thoroughly by convection throughout the FOC. In writing equations such as (4.1), we assume that the quantities concerned are averaged over a few convective overturning times, i.e., of the order of a thousand years, say.

How are motions in the Earth's core created? The obvious answer is that radioactive elements dissolved in the core, heat the fluid and provide the buoyancy that drives thermal convection. The view of the geochemists has however hardened against this idea, and it is now widely stated that there is no significant radioactivity in the core; see, for example, Stacey (1992). Recently this view has been challenged (see §6), and we shall suppose here that radioactivity supplies energy to the core at a rate, $\mathcal{Q}^{R}$, that is not necessarily zero. We shall, nevertheless, at first accept the traditional view and set $\mathcal{Q}^{R}=0$. This does not rule out thermal convection as a source of motion. If there are no radioactive sources in the core, it must be cooling, and when bodies of fluid are cooled sufficiently rapidly they convect. 
The terms $\mathcal{Q}^{L}, \mathcal{Q}^{S}$ and $\mathcal{Q}^{G}$ are all proportional to the rate of advance of the ICB. We incorporate this effect through a parameter $\lambda$ which crudely represents the age $\tau_{\mathrm{SIC}}$ of the inner core, normalized to $1.2 \mathrm{Gyr}$, e.g., to obtain these sources for $\tau_{\mathrm{SIC}}=0.6$ Gyr, we should set $\lambda=\frac{1}{2}$ in (4.3), (4.6) and (4.9) below. More precisely, we suppose that $\dot{\mathcal{M}}_{\text {SIC }}=\mathcal{M}_{\text {SIC }} /(\lambda \times 1.2 \mathrm{Gyr})$, where $\lambda$ is constant. The radioactive source is also timedependent, decreasing exponentially with time, according to the half-life of the radioactive element concerned; though easily incorporated, we do not include that effect here.

Because of the convection, the actual heat, $\mathcal{Q}_{\mathrm{ICB}}$ and $\mathcal{Q}_{\mathrm{CMB}}$, entering and leaving the fluid core may be expected to differ from $\mathcal{H}_{\mathrm{ICB}}$ and $\mathcal{H}_{\mathrm{CMB}}$, perhaps substantially. If we take $\kappa_{M}=6 \times 10^{-6} \mathrm{~m}^{2} \mathrm{~s}^{-1}$ in the SIC, we find that its thermal time constant $r_{\mathrm{ICB}}^{2} / \kappa_{M}$ is about 6 Gyr, which suggests that $T$ is "frozen-in" to the SIC, and that $\nabla T$ is of the order of the melting point gradient, which is less than the adiabatic gradient in the overlying FOC. If this is the case, $\mathcal{Q}_{\mathrm{ICB}}$ exceeds $\mathcal{H}_{\mathrm{ICB}}$, though not by a large factor. Since however the following arguments are insensitive to the value we assume for $\mathcal{Q}_{\mathrm{ICB}}$, we shall for simplicity assume that

$$
\mathcal{Q}_{\mathrm{ICB}}=\mathcal{H}_{\mathrm{ICB}} .
$$

The rate, $\mathcal{Q}^{L}$, at which latent heat is released at the ICB is $L \dot{\mathcal{M}}_{\text {SIC }}$, which for our chosen material properties is

$$
\mathcal{Q}^{L}=4.0 / \lambda \mathrm{TW}
$$

The sources $\mathcal{Q}^{S}$ and $\mathcal{Q}^{G}$ are in general harder to estimate. Because $\alpha$ and $\Delta^{s} \rho$ are nonzero, the Earth contracts as it cools, and the CMB moves systematically inwards, releasing gravitational energy as it does so. The inward motion is associated with " $P d V$ work" that increases the internal energy of the FOC. According to Loper (1991), the energy associated with contraction is surprisingly large * , although Gubbins et al. (1979) argue that it is not useful work for driving the dynamo. Matters such as these greatly complicate the theoretical analysis of the energy and entropy budgets, and in the interests of simplicity we eliminate them. This is easily done by assuming, as Braginsky and Roberts (1995) did, that the radius, $r_{\mathrm{CMB}}$, of the $\mathrm{CMB}$ is constant. The release of gravitational energy then comes about because $\Delta^{\xi} \rho$ is nonzero; the fractionation of constituents at the ICB causes the buoyant light material released to move upwards and the heavy constituent to move downwards, i.e., the Earth is continually becoming more centrally condensed.

Considering $\mathcal{Q}^{S}$ first, we have

$$
\mathcal{Q}^{S} \equiv-\int_{\mathrm{FOC}} \dot{S}_{a} T_{a} \rho_{a} d^{3} x=-\dot{S}_{a} \int_{\mathrm{FOC}} T_{a} \rho_{a} d^{3} x=-\dot{S}_{a} \bar{T} \mathcal{M}_{\mathrm{FOC}}
$$

Braginsky and Roberts (1995) showed that, to a good approximation,

$$
\dot{S}_{a}=-\Delta_{2} c_{p} \dot{r}_{\mathrm{ICB}} / r_{\mathrm{ICB}}
$$

* His method of computing (4.6) below leads to a value of $\mathcal{Q}^{S}$ almost twice as large as ours. The difference must be attributed to contraction of the CMB, which is included in his model but not ours. 
where $\Delta_{2}$ is the constant defined in (3.16)-(3.18) above. Assuming the value $\Delta_{2}=0.038$ obtained there and substituting $\dot{r}_{\mathrm{ICB}}=\dot{\mathcal{M}}_{\mathrm{SIC}} / 4 \pi r_{\mathrm{ICB}}^{2} \rho_{\mathrm{ICB}}$, we find that (4.4) and (4.5) give

$$
\mathcal{Q}^{S}=2.3 / \lambda \mathrm{TW}
$$

Gravitational energy is released into the FOC at the rate

$$
\mathcal{Q}^{G}=-\dot{\xi}_{a} \int_{\mathrm{FOC}}\left(\mu_{a}-\mu_{\mathrm{ICB}}\right) \rho_{a} d^{3} x
$$

where $\mu$ is the chemical potential. Since $\alpha^{\xi}$ is uniform in the FOC, by assumption, we may replace $(4.7)$ by

$$
\mathcal{Q}^{G}=\dot{\xi}_{a} \alpha^{\xi} \int_{\mathrm{FOC}}\left(U_{\mathrm{ICB}}-U_{a}\right) \rho_{a} d^{3} x=\dot{\xi}_{a} \alpha^{\xi} \mathcal{E}_{\mathrm{FOC}}
$$

where (3.7) and (3.15) provide $\mathcal{E}_{\mathrm{FOC}}$ and $\alpha^{\xi}$. Since $\dot{\xi}_{a}=4 \pi r_{\mathrm{ICB}}^{2} \dot{r}_{\mathrm{ICB}}^{2} \rho_{\mathrm{SIC}} \Delta \xi / \mathcal{M}_{\mathrm{FOC}}$ (see Braginsky and Roberts 1995), it follows that

$$
\mathcal{Q}^{G}=0.5 / \lambda \mathrm{TW}
$$

Collecting together our results, we have

$$
\mathcal{Q}_{\mathrm{CMB}}=\frac{6.8 \mathrm{TW}}{\lambda}+0.3 \mathrm{TW}+\mathcal{Q}^{R}
$$

The material properties assumed by Braginsky and Roberts (1995) imply values of the constants featuring in (4.3), (4.6) and (4.9) that are substantially different from ours; they were 2.5, 3.1 and 1.3, respectively. But these values give almost the same result as (4.10); the 6.8 appearing in (4.10) was replaced by 6.9 , so that $\mathcal{Q}^{R}=0$ and $\mathcal{Q}_{\mathrm{CMB}}=7.2 \mathrm{TW}$, which were the values assumed by Glatzmaier and Roberts (1996a) in their simulation, imply that $\lambda=1$, i.e., $\tau_{\mathrm{SIC}}=1.2$ Gyr. Not surprisingly, though perhaps comfortingly, the rate of advance of the ICB in the simulation had already indicated that $\tau_{\mathrm{SIC}} \approx 1.2 \mathrm{Gyr}$. Despite the similarity of the two estimates for $\mathcal{Q}_{\mathrm{CMB}}(7.1 \mathrm{TW}$ and $7.2 \mathrm{TW})$, the gross thermodynamics of the two models are rather different, as is indicated below.

If $\tau_{\mathrm{SIC}}=4$ Gyr and $\mathcal{Q}^{R}=0,(4.10)$ gives $\mathcal{Q}_{\mathrm{CMB}}=2.3 \mathrm{TW}$, which is less than a half of the adiabatic heat flux $\mathcal{H}_{\mathrm{CMB}}$. As Loper (1978) pointed out, the superadiabatic heat flow at the CMB, which is

$$
\mathcal{Q}_{\mathrm{sa}} \equiv \mathcal{Q}_{\mathrm{CMB}}-\mathcal{H}_{\mathrm{CMB}}=\left(\frac{6.8}{\lambda}-5.6\right) \mathrm{TW}
$$

may in principle be either positive or negative. It is not impossible, as Glatzmaier and Roberts (1997) show through a concrete example, for a dynamo with a subadiabatic $\mathcal{Q}_{\mathrm{CMB}}$ to function, but it is unlikely that such a dynamo would be vigorous enough to undergo polarity reversals. This illustrates the difficulty in supposing that the solid inner core is an old feature of the Earth. 
The rate of working of the buoyancy forces does not feature in (4.1), since the kinetic and magnetic energies buoyancy creates is returned to the core as heat through ohmic and viscous dissipation.

\subsection{Entropy balance}

The energy balance does not suffice to describe the gross state of the core completely. The entropy balance, which depends on the available dissipation, adds a further constraint. This was first recognized by Braginsky (1964). The subject was advanced by Backus (1975), Hewitt et al. (1975), Gubbins et al. (1979), Braginsky and Roberts (1995) and others.

The entropy balance of the FOC is expressed by

$$
\frac{\mathcal{Q}_{\mathrm{CMB}}}{T_{\mathrm{CMB}}}=\frac{\mathcal{Q}_{\mathrm{ICB}}+\mathcal{Q}^{L}}{T_{\mathrm{ICB}}}+\Sigma+\frac{\mathcal{Q}^{D}}{T_{D}}+\frac{\mathcal{Q}^{S}+\mathcal{Q}^{R}}{\bar{T}},
$$

where $\mathcal{Q}_{\mathrm{ICB}} / T_{\mathrm{ICB}}$ and $\mathcal{Q}_{\mathrm{CMB}} / T_{\mathrm{CMB}}$ are the entropy flows in and out of the boundaries, and $\mathcal{Q}^{L} / T_{\mathrm{ICB}}$ is the entropy released at the SIC through freezing. The remaining terms are volumetric sources within the FOC and are discussed more fully below.

Entropy is produced volumetrically through the diffusion of field, vorticity, heat and composition. Of these, the most significant is $\Sigma$, which arises from heat conduction down the adiabatic gradient and which is evaluated in (3.11). In calculating the remaining diffusive contributions, we adopt the two-scale strategy of $\S 1$. Because $R e, P e$ and $M p$ are large, the entropy production per unit volume by the macroscale is dominated by $\bar{\sigma}=q^{\bar{J}} / T_{a}$, where $q^{\bar{J}}=\mu_{0} \eta_{M} \overline{\mathbf{J}}^{2}$ is the macroscopic dissipation rate, see $\S 1$. The entropy production by thermal and compositional diffusion is dominated by turbulent diffusion, through the fluxes $\mathbf{I}^{S} \equiv \overline{S^{\prime} \mathbf{V}^{\prime}}$ and $\mathbf{I}^{\xi} \equiv \overline{\xi^{\prime} \mathbf{V}^{\prime}}$ where, according to the Reynolds analogy, $\mathbf{I}^{S}=-\rho_{a} \kappa_{T} \nabla \bar{S}$ and $\mathbf{I}^{\xi}=-\rho_{a} \kappa_{T} \nabla \bar{\xi}$; see $\S 1$. Braginsky and Roberts (1995) showed in $\S 4.2$ and Appendix $\mathrm{C}$ of their paper that the resulting mean entropy production could be written as $\sigma^{\prime}=-\mathbf{g}_{a} \cdot\left(\alpha^{S} \mathbf{I}^{S}+\alpha^{\xi} \mathbf{I}^{\xi}\right) / T_{a}$, i.e., as

$$
\sigma^{\prime}=\rho_{a} \kappa_{T} \mathbf{g}_{a} \cdot\left(\alpha^{S} \nabla \bar{S}+\alpha^{\xi} \nabla \bar{\xi}\right) / T_{a},
$$

an expression that they related to the average rate at which buoyancy feeds energy into the microscale to maintain the turbulence. This form of $\sigma^{\prime}$ is useful in numerical computations, and was adopted by Glatzmaier and Roberts (1996), who used it to obtain the estimate $\mathcal{Q}^{J^{\prime}}=0.1 \mathrm{TW}$ mentioned in $\S 2.1$ (and there criticised as being too small).

Braginsky and Roberts (1995) also showed that the mean entropy production by the microscale could be written as $\sigma^{\prime}=q^{J^{\prime}} / T_{a}$ per unit volume, where $q^{J^{\prime}}=\mu_{0} \eta_{M} \overline{\left(\mathbf{J}^{\prime}\right)^{2}}$ is the average microscopic ohmic dissipation, see $\S 1$. At first sight, this result may seem surprising. One way of rationalizing it is to imagine oneself moving forward in time along a hypothetical sequence at the end point of which, in the remote future, computer technology has developed to the point where $\mathbf{V}, \mathbf{B}, S$ and $\xi$ are fully resolved, including the parts we have called $\mathbf{V}^{\prime}, \mathbf{B}^{\prime}, S^{\prime}$ and $\xi^{\prime}$. The length and time scales become increasingly well resolved along this sequence, the turbulent viscosity $\nu_{T}$ and the turbulent diffusivity $\kappa_{T}$ of heat and composition become increasingly irrelevent, and finally only the molecular diffusivities $\nu_{M}, \kappa_{M}$ and $D_{M}$ act. Because these are small compared with $\eta_{M}$, their 
contributions to the entropy production will then be negligible compared with the ohmic rate of entropy production. And this, because it now is calculated from electric currents of all length scales, has increased to what, according to the two-scale ansatz, has been written as $\left(q^{\bar{J}}+q^{J^{\prime}}\right) / T_{a}=q^{J} / T_{a}$. In short, the entropy production is the same at one end of our hypothetical sequence as the other, but it is partitioned differently. Initially, it is (4.13) but finally it is what we have called $q^{J^{\prime}} / T_{a}$.

In recognition of the fact that Joule losses dominate the Dissipative processes, we write $q^{\bar{J}}+q^{J^{\prime}}$ as $q^{D}$ rather than $q^{J}$. Integrating $q^{D}$ over the FOC, we obtain a net dissipation rate of

$$
\mathcal{Q}^{D}=\int_{\mathrm{FOC}} q^{D} d^{3} x
$$

and in (4.12) we have, in the interests of simplicity, written the net entropy production rate as

$$
\frac{\mathcal{Q}^{D}}{T_{D}}=\int_{\mathrm{FOC}} \frac{q^{D}}{T_{a}} d^{3} x
$$

The estimates of $\mathcal{Q}^{J}$ made in $\S 2$ contain all the information about $\mathcal{Q}^{D}$ that we require.

It is clear that $T_{\mathrm{CMB}}<T_{D}<T_{\mathrm{ICB}}$ and, since the superadiabatic gradient is almost certainly largest near the bottom of the FOC (leading to more active convection, field generation and associated dissipation), it is plausible that $T_{D}>\bar{T}$. The Glatzmaier and Roberts simulations tend to show strong activity within and deep in the tangent cylinder and, although currents generated in these regions leak out into the exterior of the tangent cylinder, the main Joule heat losses appear to be within it, again suggesting that $T_{D}>\bar{T}$. It is possible in principle to estimate $T_{D}$ from a dynamo simulation, but in practice the hyperdiffusion demanded for numerical reasons makes such estimates unreliable.

The entropy source associated with the radiogenic heat sources in the FOC may be similarly written as

$$
\frac{\mathcal{Q}^{R}}{T_{R}}=\int_{\mathrm{FOC}} \frac{q^{R}}{T_{a}} d^{3} x
$$

Since these sources are thoroughly mixed by the convection, the rate $q^{R} / \rho_{a}$ at which they inject energy is presumably uniform over the FOC, so that $T_{R}=\widetilde{T}$. Here $1 / \widetilde{T}$ is the mass weighted average of $1 / T_{a}$, which was evaluated in $\S 3.2$. In what follows we shall, for simplicity, take $T_{R}=\bar{T}$.

The entropy source associated with the loss of internal energy through the cooling of the Earth is

$$
\int_{\mathrm{FOC}} \dot{S}_{a} \rho_{a} d^{3} x=\mathcal{M}_{\mathrm{FOC}} \dot{S}_{a}=-\frac{\mathcal{Q}^{S}}{\bar{T}}
$$

by (4.4).

According to (4.1), (4.2) and (4.12), we now have

$$
\mathcal{Q}^{D}=\mathcal{Q}^{G}+\mathcal{Q}^{H}
$$


where $\mathcal{Q}^{H}$ is the part of $\mathcal{Q}^{D}$ available from Heat sources:

$$
\begin{gathered}
\mathcal{Q}^{H}=\mathcal{Q}_{\mathrm{CMB}}\left(\frac{T_{D}}{T_{\mathrm{CMB}}}-1\right)+\left(\mathcal{H}_{\mathrm{ICB}}+\mathcal{Q}^{L}\right)\left(1-\frac{T_{D}}{T_{\mathrm{ICB}}}\right) \\
+\left(\mathcal{Q}^{S}+\mathcal{Q}^{R}\right)\left(1-\frac{T_{D}}{\bar{T}}\right)-\Sigma T_{D} .
\end{gathered}
$$

Thus, as anticipated by Braginsky (1964), all the gravitational energy released is available for dissipation but only a part, proportional to $\Delta T / T$ for some $\Delta T$, of each thermal contribution to $\mathcal{Q}^{D}$ can be used; in the case of $\Sigma$, this is evident from its definition (3.11).

This emasculation of the heat sources is reminiscent of the heat engines of classical thermodynamics, and in particular of the Carnot cycle. In such cases, $\mathcal{Q}^{D}$ might, since it relates to the creation of the observed geomagnetic field, be called "useful work", but such a description is inappropriate when this energy is unavailable outside the system but is re-deposited within it. The term "available dissipation" may be preferable.

In $\S 4.3$ we shall make use of (4.1) and (4.2) to write (4.18) and (4.19) as

$$
\mathcal{Q}^{D}=\frac{T_{D}}{T_{\mathrm{CMB}}}\left[\left(\mathcal{H}_{\mathrm{ICB}}+\mathcal{Q}^{L}\right)\left(1-\frac{T_{\mathrm{CMB}}}{T_{\mathrm{ICB}}}\right)+\left(\mathcal{Q}^{S}+\mathcal{Q}^{R}\right)\left(1-\frac{T_{\mathrm{CMB}}}{\bar{T}}\right)+\mathcal{Q}^{G}-\Sigma T_{\mathrm{CMB}}\right] .
$$

Using the constants we have derived above, we may write this as

$$
\mathcal{Q}^{D}=\frac{T_{D}}{T_{\mathrm{CMB}}}\left(\frac{1.69 \mathrm{TW}}{\lambda}-0.62 \mathrm{TW}+0.12 \mathcal{Q}^{R}\right) .
$$

Let us suppose (until $\S 6$ ) that $\mathcal{Q}^{R}=0$. Then it is clear from $(4.21)$ that, since $\mathcal{Q}^{D}$ must be positive, $\lambda<2.7$, i.e., $\tau_{\text {SIC }}<3.3 \mathrm{Gyr}$. This crude estimate is refined if we place $\mathcal{Q}^{D}$ in the range (2.19). We examine two possibilities, case (a) modelling a dynamo where the Joule heat loss is uniform over the core and case (b) modelling a dynamo where the dissipation occurs preferentially nearer the ICB:
(a) $T_{D}=\bar{T}=4488^{\circ} \mathrm{K}$,
(b) $T_{D}=\frac{1}{2}\left(\bar{T}+T_{\mathrm{ICB}}\right)=4794^{\circ} \mathrm{K}$.

We then find that, corresponding to $1 \mathrm{TW}<\mathcal{Q}^{D}<2 \mathrm{TW}$, we have

$$
\begin{array}{lll}
\text { (a) } & 6.3 \mathrm{TW}<\mathcal{Q}_{\mathrm{CMB}}<9.9 \mathrm{TW}, & 1.34 \mathrm{Gyr}>\tau_{\mathrm{SIC}}>0.85 \mathrm{Gyr}, \\
\text { (b) } & 6.1 \mathrm{TW}<\mathcal{Q}_{\mathrm{CMB}}<9.4 \mathrm{TW}, & 1.40 \mathrm{Gyr}>\tau_{\mathrm{SIC}}>0.89 \mathrm{Gyr} .
\end{array}
$$

Let us define the 'critical dynamo' as one in which the Nusselt number, $N u \equiv$ $\mathcal{Q}_{\mathrm{CMB}} / \mathcal{H}_{\mathrm{CMB}}$, is 1 . According to (4.10) and (4.21), this corresponds to $\tau_{\mathrm{SIC}}=1.46$ Gyr (and $\mathcal{Q}^{D}=0.87 \mathrm{TW}$ ). Subcritical dynamo action is not impossible, as was established by a model of Glatzmaier and Roberts (1997), but that dynamo could only produce a field small compared with the Earth's, one that gave no indications of reversing. While other factors are involved, such as the way the flow of heat $\mathcal{Q}_{\mathrm{CMB}}$ out of the core is partitioned over the CMB (Glatzmaier et al. 1999), numerical simulations suggest that the larger the 
value of $N u$ the greater the frequency of reversals. The upper bounds in (4.22) correspond to $N u \approx 1.7$. This may be compared with $N u$ for the 8 models studied by (Glatzmaier et al. 1999), which was only about 1.3 even though 7 of the model reversed (some frequently). Probably, the upper bounds on $\mathcal{Q}_{\mathrm{CMB}}$ in (4.22) correspond to dynamos that reverse fairly frequently, while the lower bounds correspond to slightly supercritical dynamos that are likely to be weak and nonreversing. The thermodynamic arguments we present here suggest that the distinction between these two types of behavior is better made on the basis of $\mathcal{Q}^{D}$ than on the basis of $N u$.

A striking feature of (4.22) is the youth of the inner core, even when the upper bounds for $\tau_{\text {SIC }}$ are assumed. This raises the question of how the geodynamo functioned early in the Earth's history, when the sources of latent heat and light component at the ICB were absent. We discuss this briefly in $§ 4.4$.

\subsection{Variants}

This subsection explores the effect of changing each of the four most uncertain parameters: the contraction on solidification, $\Delta^{s} V$; the depression of the melting point through alloying, $T_{m}$; the mass fraction of the light component, $\xi_{a}$; and the heat of reaction $h^{\xi}$. Three of the values

$$
\Delta^{s} V=2 \times 10^{-6} \mathrm{~m}^{3} \mathrm{~kg}^{-1}, \quad T_{m}=300^{\circ} \mathrm{K}, \quad \xi_{a}=0.095, \quad h^{\xi}=-1.6 \times 10^{7} \mathrm{~J} \mathrm{~kg}^{-1},
$$

are held fixed as the fourth is changed. The results are shown in Tables 1-4, where it is supposed throughout that the melting point of pure iron is $5400^{\circ} \mathrm{K}$. The values of $\mathcal{Q}^{L}, \mathcal{Q}^{S}$ and $\mathcal{Q}^{G}$ assume that the age of the inner core is $\tau_{\mathrm{SIC}}=1.2 \mathrm{Gyr}$; if instead it is $1.2 \lambda \mathrm{Gyr}$, the values should be divided by $\lambda$. We also write the energy and entropy balances as

$$
\begin{aligned}
& \mathcal{Q}_{\mathrm{CMB}}=\frac{E_{\lambda}}{\lambda}+\mathcal{H}_{\mathrm{ICB}}+\mathcal{Q}^{R}, \\
& \mathcal{Q}^{D}=\frac{T_{D}}{T_{\mathrm{CMB}}}\left(\frac{D_{\lambda}}{\lambda}-D_{0}+0.12 \mathcal{Q}^{R}\right) .
\end{aligned}
$$

We have here given $1-T_{\mathrm{CMB}} / \bar{T}$ the value 0.12 that it has in every case considered here.

It is clear from Table 1 that, as anticipated in $\S 3.4$, many results are sensitive to $\Delta^{s} V$. Table 2 shows that $T_{\mathrm{ICB}}, T_{\mathrm{CMB}}$, and $\bar{T}$ are very dependent on $T_{m}$; also $\mathcal{H}_{\mathrm{ICB}}, \mathcal{H}_{\mathrm{CMB}}$ and $D_{0}$ vary by about a factor of 2 across the range shown but other quantities, including $\mathcal{Q}^{L}$, $\mathcal{Q}^{S}, E_{\lambda}$ and $D_{\lambda}$, are less affected. The constants appearing in (4.24) and (4.25) are also fairly insensitive to $\xi_{a}$ and $h^{\xi}$, as is shown in Tables 3 and 4 .

In $\S 4.2$, we explored relations (4.10) and (4.21) for our 'standard model' (4.23) in three ways. First, we used them to set a crude upper limit on the age of the inner core, $\tau_{\mathrm{SIC}}$; second we used the upper and lower bounds in (2.19) to limit $\tau_{\mathrm{SIC}}$ and to calculate the corresponding $\mathcal{Q}_{\mathrm{CMB}}$; third, we supposed that the Nusselt number, $N u \equiv \mathcal{Q}_{\mathrm{CMB}} / \mathcal{H}_{\mathrm{CMB}}$ was unity and determined $\tau_{\mathrm{SIC}}$ for that 'critical state', together with the corresponding value of $\mathcal{Q}^{D}$. In all cases $\mathcal{Q}^{R}$ was assumed to be zero. Since the differences between (4.22a) and $(4.22 \mathrm{~b})$ were not substantial, we confine attention here to case (a), in which $T_{D}=\bar{T}$. Tables 1-4 show the corresponding results for all the models considered. The crude limit is denoted by $\tau_{\text {SIC }}^{\text {crude }}$; values for the lower limit in $(4.22)$ corresponding to $\mathcal{Q}^{D}=1 \mathrm{TW}$ carry 
a superfix ${ }^{1}$ and those for the upper limit for $\mathcal{Q}^{D}=2$ TW carry the superfix ${ }^{2}$; values denoted by $\tau_{\text {SIC }}^{\mathrm{c}}$ and $\mathcal{Q}_{\mathrm{c}}^{D}$ refer to the critical case $N u=1$. Perhaps the most striking fact that emerges from the tabulated values is by how little they vary, even for the different values of $\Delta^{s} V$. In particular, all the upper bounds $\tau_{\text {SIC }}^{1}$ of $\tau_{\text {SIC }}$ are considerably less than the known age of the geomagnetic field (Kono and Tanaka 1995).

\subsection{The early Earth}

It was mentioned in $\S 4.1$ that the Glatzmaier and Roberts (1996a) model was consistent with an inner core that is only 1.2 Gyr old. Other research has led to similar estimates of $\tau_{\text {SIC }}$. Stevenson et al. (1983) estimated that $\tau_{\text {SIC }} \lesssim 2.3$ Gyr, Labrosse et al. (1997) that $\tau_{\text {SIC }} \lesssim 1.7$ Gyr and Buffett et al. (1996) that $\tau_{\text {SIC }} \approx 2.8$ Gyr. But it is known that the geomagnetic field has existed, with an amplitude within a factor of 4 of its present strength, for at least 3.5 Gyr; see Kono and Tanaka (1995). It is of interest to consider how the estimates of $\S 4.2$ have to be modified prior to the formation of the inner core.

In looking at this question in slightly greater depth, we make some simplifications. The present SIC is small compared with the FOC, both in terms of mass and volume, and this was even more true in the past. We therefore trust that we are not making a serious error by adopting the same value (2.11) of $\Sigma$ as before, and by taking the same values (2.9) of $T_{\mathrm{CMB}}$ and $\bar{T}$. Of course, prior to the birth of the inner core, $\mathcal{Q}^{L}=\mathcal{Q}^{G}=\mathcal{H}_{\mathrm{ICB}}=0$, and the dynamo is entirely thermally-driven. Then (4.1) and (4.12) are replaced by

$$
\begin{aligned}
& \mathcal{Q}_{\mathrm{CMB}}=\mathcal{Q}^{S}+\mathcal{Q}^{R}, \\
& \frac{\mathcal{Q}_{\mathrm{CMB}}}{T_{\mathrm{CMB}}}=\Sigma+\frac{\mathcal{Q}^{D}}{T_{D}}+\frac{\mathcal{Q}^{S}+\mathcal{Q}^{R}}{\bar{T}} .
\end{aligned}
$$

The first of these is no more than a convenient way of defining the rate of cooling.

We may write (4.26) and (4.27) in the forms (4.24) and (4.25), with $\mathcal{H}_{\mathrm{ICB}}$ set zero and different values of the constants $E_{\lambda}, D_{0}$ and $D_{\lambda}$. In our standard model, we have

$$
\begin{aligned}
& \mathcal{Q}_{\mathrm{CMB}}=\frac{2.31 \mathrm{TW}}{\lambda}+\mathcal{Q}^{R} \\
& \mathcal{Q}^{D}=\frac{T_{D}}{T_{\mathrm{CMB}}}\left(\frac{0.28 \mathrm{TW}}{\lambda}-0.68 \mathrm{TW}+0.12 \mathcal{Q}^{R}\right) .
\end{aligned}
$$

The parameter $\lambda$ has, of course, a different interpretation than in $\S \S 4.1-4.3$, where it referred to the growth rate of the inner core. In (4.28) and (4.29), $1.2 \lambda$ is the timescale, $\tau_{\text {cooling, }}$, over which the fluid core cools.

Taking $\mathcal{Q}^{R}=0$ and $T_{D}=\bar{T}$ as before, we find that $\tau_{\text {cooling }}=0.49 \mathrm{Gyr}$, and that the limits (2.19) give

$$
12.9 \mathrm{TW}<\mathcal{Q}_{\mathrm{CMB}}<20.1 \mathrm{TW}, \quad 0.22 \mathrm{Gyr}>\tau_{\text {cooling }}>0.14 \text { Gyr } .
$$

The other values of $\xi_{a}$ explored in Table 3 give similar results. The small limits on $\tau_{\text {cooling }}$ given in (4.30) are not encouraging for the success of the dynamo when the inner core is absent; primordial heat must be lost quickly to drive a dynamo of the required strngth and whether the mantle is able to transmit so much heat is uncertain. 


\section{THERMODYNAMIC EFFICIENCY}

There is no unique definition of the efficiency of the geodynamo, considered as a heat engine. Nevertheless, it is tempting to define the useful work to be the part $\mathcal{Q}^{\overline{\mathcal{J}}}$ of $\mathcal{Q}^{D}$ that can be estimated through geodynamo simulations. Then the efficiency of the Dynamo is

$$
\epsilon_{D} \equiv \frac{\mathcal{Q}^{\overline{\mathcal{J}}}}{\mathcal{Q}_{\mathrm{CMB}}}
$$

which we write as

$$
\epsilon_{D}=f_{F} \epsilon_{G},
$$

where $\epsilon_{G}$ is the ideal Geodynamo efficiency,

$$
\epsilon_{G} \equiv \frac{\mathcal{Q}^{D}}{\mathcal{Q}_{\mathrm{CMB}}}
$$

and

$$
f_{F}=\frac{\mathcal{Q}^{\overline{\mathcal{J}}}}{\mathcal{Q}^{D}}
$$

is a "frictional factor". By (4.19), we therefore have (taking $\mathcal{Q}^{R}=0$ and $T_{D}=\bar{T}$ as before)

$$
\epsilon_{G}=\left(\frac{\bar{T}}{T_{\mathrm{CMB}}}-1\right)+\frac{1}{\mathcal{Q}_{\mathrm{CMB}}}\left[-\Sigma \bar{T}+\mathcal{Q}^{G}+\left(\mathcal{H}_{\mathrm{ICB}}+\mathcal{Q}^{L}\right)\left(1-\frac{\bar{T}}{T_{\mathrm{ICB}}}\right)\right] .
$$

Our estimates of $\S 4$ give (for $\lambda=1$ )

$$
\epsilon_{G} \approx 17 \%, \quad \epsilon_{D} \approx 4 \%, \quad f_{F} \approx 0.25
$$

A similar conclusion follows from an expression* derived by Braginsky and Roberts (1995):

$$
\epsilon_{G} \approx \frac{1}{\mathcal{Q}_{\mathrm{CMB}}}\left[\mathcal{Q}^{G}+\left(\frac{\bar{T}}{T_{\mathrm{CMB}}}-1\right)\left(\mathcal{Q}_{\mathrm{sa}}+\mathcal{H}_{\mathrm{ICB}}+\mathcal{Q}^{L}\right)\right]
$$

this is about $18 \%$. Equation (5.7) gives full force to the gravitation source but the two remaining (thermal) sources are each diminished in usefulness by the factor $\left(\bar{T} / T_{\mathrm{CMB}}-1\right) \approx$ 0.14 .

It was remarked in $\S 4.1$ that the parameter values of Braginsky and Roberts (1995) gave much the same $\mathcal{Q}_{\mathrm{CMB}}$ as here, but that the individual contributions to $\mathcal{Q}_{\mathrm{CMB}}$ were different. Here the thermal driving is larger and the dynamo is therefore less efficient;

* Their result followed from the energy and entropy balances for the whole core using their modified Boussinesq theory. It also depended on a coincidence which makes it inappropriate for $\S 6$ below: in their model, $2\left(1-T_{\mathrm{CMB}} / \bar{T}\right) \approx 1-T_{\mathrm{CMB}} / T_{\mathrm{ICB}}$. We use balances for the FOC alone, and have therefore again allowed for the fluxes across the ICB by replacing $\mathcal{Q}^{L}$ by $\mathcal{Q}^{L}+\mathcal{Q}_{\mathrm{CMB}} \approx \mathcal{Q}^{L}+\mathcal{H}_{\mathrm{CMB}}$. To obtain (5.7) from their expression, we write $T_{D}=\bar{T}$, as before. 
in the case of Braginsky and Roberts (1995), both (5.6) and (5.7) gave $\epsilon_{G} \approx 28 \%$ and $f_{F} \approx 0.15$.

When there is no inner core, $(5.5)$ reduces (for $\mathcal{Q}^{R}=0$ and $T_{D}=\bar{T}$ ) to

$$
\epsilon_{G}=\left(\frac{\bar{T}}{T_{\mathrm{CMB}}}-1\right)-\frac{\Sigma \bar{T}}{\mathcal{Q}_{\mathrm{CMB}}}
$$

For order of magnitude purposes, we take $\Sigma \bar{T} / \mathcal{Q}_{\mathrm{CMB}}=0.11$ as before, and obtain

$$
\epsilon_{G} \approx 3 \%
$$

which confirms the difficulty faced when the inner core is absent and there is no radiactivity.

\section{THE RADIOACTIVE CORE}

\subsection{Where is the potassium?}

As the Sun contains $99.9 \%$ of the mass in the solar system, it is reasonable to suppose that its composition resembles that of the early planetary nebula. In confirmation, it has been observed that the CI chondrite meteoritic class, which represents the most primitive of all the undifferentiated meteorites, is unique in having a composition close to that of the Sun (Grevesse et al. 1996). It has long been argued the Earth's composition is broadly chondritic (e.g., Ringwood 1959, 1975, 1979; Wanke 1981; Sun 1982; Wasson and Kallemeyn 1988), at least with respect to the refractory elements. Conventional wisdom holds that, because of the progressive decrease in the average mid-plane temperature of the nebula with increasing heliocentric distance, volatile elements in gaseous phase near the Sun would evaporate and be blown outwards by the solar wind, which was particularly violent during the early, T-Tauri stage of the Sun's evolution. This explains the change from the rocky inner planets (Mercury, Venus, Earth, Mars) to the giant gaseous outer planets (Jupiter, Saturn, Neptune, Uranus). For this reason, most modern workers would argue that the Earth's composition is that of a devolatilized CI chondrite (e.g., Hart and Zindler 1986, 1989; McDonough and Sun 1995).

This idea is consistent with estimates of the concentrations of uranium, thorium and potassium in the Bulk Silicate Earth (BSE), the name given to the homogeneous reservoir representing the sum of the continental crust, continental lithosphere and the depleted mantle reservoirs, which taken together and appropriately weighted give

$$
\mathrm{U}_{\mathrm{BSE}}=11.92 \mathrm{ppb}, \quad \mathrm{Th}_{\mathrm{BSE}}=46.13 \mathrm{ppb}, \quad \mathrm{K}_{\mathrm{BSE}}=140 \mathrm{ppm} .
$$

$(\mathrm{ppb}=$ parts per billion; $\mathrm{ppm}=$ parts per million. $)$

It is generally agreed that $\mathrm{U}$ and Th cannot exist in the core and, if the same is true for K, we may translate (6.1) into concentrations for the Bulk Earth (BE), i.e., the Earth as a whole, by multiplying the entries by $\omega=0.670$, the ratio of the mass of the BSE to that of the entire Earth:

$$
\mathrm{U}_{\mathrm{BE}}=8.0 \mathrm{ppb}, \quad \mathrm{Th}_{\mathrm{BE}}=30.9 \mathrm{ppb}, \quad \mathrm{K}_{\mathrm{BE}}=93.7 \mathrm{ppm} .
$$


These figures may be compared with the average CI chondrite values (e.g., McDonough and Sun 1995):

$$
\mathrm{U}_{\mathrm{CI}}=8.1 \mathrm{ppb}, \quad \mathrm{Th}_{\mathrm{CI}}=28.9 \mathrm{ppb}, \quad \mathrm{K}_{\mathrm{CI}}=555 \mathrm{ppm} .
$$

The U/Th ratios are very similar: 0.26 for $\mathrm{BE}$ and 0.28 for CI, which are well within the uncertainties of estimation, but the $\mathrm{K} / \mathrm{U}$ ratios are very different: $1.2 \times 10^{4}$ for a potassium depleted BE but $6.7 \times 10^{4}$ for CI.

There are two extreme explanations for this fact, and any number of intermediate possibilities. One extreme is the traditional view, which is today widely held by the geochemical and cosmochemical community: potassium is a moderately volatile element and the missing $\mathrm{K}$ was lost during the formation of the Earth. The other extreme is the unorthodox view that the missing $\mathrm{K}$ resides in the core.

The traditional view depends on the belief that equilibrium liquid silicate/liquid metal partitioning forbids any $\mathrm{K}$ from entering the core. This view is currently so strongly held that it is used as a way of placing a lower bound of $1100^{\circ} \mathrm{K}$ on the temperature of the solar nebula at the time of Earth's formation, this being the $50 \%$ condensation temperature of K (Larimar 1988; Palme et al. 1988). The unorthodox view, which at one time was the traditional view (e.g., Murthy and Hall 1970, 1972; Lewis 1971; Goettel 1972, 1974, 1976), was abandoned because it was argued, mostly on the basis of low pressure partitioning experiments (e.g., Goettel 1972; Somerville and Ahrens 1980; Ito et al. 1993; Chabot and Drake 1999) that K cannot form a stable alloy with Fe. Without contesting this, Calderwood (2000) has pointed out that potassium can enter the core by another channel, as explained in the next subsection.

The traditional view rules out the presence of ${ }^{40} \mathrm{~K}$ as a heat source for the dynamo, leading to the difficulties outlined in $\S 5$.

\subsection{The magma ocean: the metallization of Potassium}

Planetary accretion modelers visualize that, after the proto-Earth reaches $\frac{1}{3}-\frac{1}{2}$ of its present mass, heating by the impact of planetesimals will create a hydrous global magma ocean (HGMO); see Davies (1985, 1990). Subsequently, as the Earth cooled, mantle-core segregation took place at the base of the HGMO under the conditions of very high temperatures and pressures prevailing there (e.g., Li and Agee 1996, 1997; Righter and Drake 1996, 1997a, 1997b, 1999; Suzuki et al. 1996; Righter et al. 1997, 2000; Thibault and Walter 1995; Wade and Wood, 2001). In this model liquid iron undergoes gravitational separation from the liquid silicate magma and ponds at the base of the magma ocean. Then, via percolation through the underlying solid mantle (e.g., Shannon and Agee 1998), this liquid metal continues to migrate deeper into the Earth. This model is invoked to explain the observed mantle depletions in $\mathrm{Co}, \mathrm{Ni}, \mathrm{W}, \mathrm{P}, \mathrm{V}, \mathrm{S}, \mathrm{Pb}, \mathrm{Ga}$, and $\mathrm{Nb}$ within the BSE relative to CI chondritic values, the argument being that the present day mantle abundancies do not represent true depletions but instead record equilibrium element partitioning during mantle-core segregation (see above references). To obtain present day BSE concentrations of these elements under equilibrium partitioning, the prevailing pressures and temperatures would have to be about

$$
P_{\mathrm{HGMO}}=22 \mathrm{GPa}, \quad T_{\mathrm{HGMO}}=2,200^{\circ} \mathrm{K},
$$


the former corresponding roughly to the bottom of the present Earth's transition zone; see Righter and Drake (1999).

The unorthodox view of Calderwood (2000) rests on the experimental partitioning coefficients of Ohtani et al. (1997). Their experiments included nickel in the metal fraction, and went to a high enough pressure $(20 \mathrm{GPa})$ to exceed comforatbly the pressure $(P>11.5 \mathrm{Gpa})$ at which $\mathrm{K}$ has been experimentally observed to undergo an s-to-d electronic transition, termed "metallization" (e.g., Winzenick et al. 1994). This change in the electronic configuration of $\mathrm{K}$ is critical because it has been shown in separate diamond anvil experiments that, once $\mathrm{K}$ has undergone metallization, it will form a stable metal alloy with Ni (Parker et al. 1996); but at pressures beneath this pressure interval, no stable alloy forms. Parker et al. (1996) point out that their results are supported by empirical chemical rules, termed Miedema Rules (Miedema et al. 1980), that explain the formation of transition metal alloys and the likelihood of alloy formation with increasing pressure. This is in sharp contrast to a possible potassium-iron metal alloy, which is neither observed at low to high pressures (e.g., Chabot and Drake 1999; Ito et al. 1993), nor is predicted to form according to the Miedema Rules (Parker et al. 1996; see also Bukowinski 1976; Liu 1986; Sherman 1990). In short, both theory and diamond anvil experiments show that potassium will readily form a high pressure alloy with nickel, but not with iron.

With the exception of Ohtani et al. (1997), previous high partitioning experiments designed to test if potassium might partition into the core did not include nickel in the starting metal phase and instead concentrated solely on either pure iron or FeS mixtures for the metal phase. Thus, it is not surprising that these experiments found very little partitioning of $\mathrm{K}$ into the metal phase.

The unorthodox view evades a difficulty encountered by the traditional view. If (see above) the solar nebula at $1 \mathrm{au}$ had been at $1100^{\circ} \mathrm{K}$, not only should the $\mathrm{K}$ have been depleted but also the ${ }^{41} \mathrm{~K} /{ }^{39} \mathrm{~K}$ isotopic ratio should be been increased relative to the incoming planetesimals (assumed of chondritic composition), and such an effect has never been observed (Humayun and Clayton 1995a,b). Furthermore, a temperature of $1100^{\circ} \mathrm{K}$ would predict an Earth even more depleted in elements that are more volatile than potassium, such as $\mathrm{Pb}$, for which the $50 \%$ condensation temperature is $525^{\circ} \mathrm{K}$ and $\mathrm{S}$ for which it is $650^{\circ} \mathrm{K}$; see Boss (1998).

\subsection{Some consequences of potassium in the core}

We pursue the unorthodox view. If we put all the missing $\mathrm{K}$ into the core, its concentration would, according to $(6.2)$ and $(6.3)$, be $(555-93.7 \omega) /(1-\omega) \approx 1420 \mathrm{ppm}$. The $1.9510^{24} \times$ $1.42010^{-3} \approx 2.82 \times 10^{21} \mathrm{~kg}$ of $\mathrm{K}$ in the core would, through the ${ }^{40} \mathrm{~K}$ it contains, generate $3.391 \times 10^{-9} \mathrm{~W} / \mathrm{kg}$ of heat, or about $9.34 \mathrm{TW}$ in total. The half-life of ${ }^{40} \mathrm{~K}$ is $1.2778 \mathrm{Gyr}$, so that its heat production 4.5 billion years ago would be 12 times as great. We at first ignore this fact.

A source of radioactivity in the core can slow down, halt, or even reverse the growth of the inner core; this depends entirely on $\mathcal{Q}_{\mathrm{CMB}}$, the heat flux from the core into the mantle, and this in turn depends on the effectiveness of mantle convection in transporting heat. The mantle is the "valve" that controls magnetoconvection in the core and the geodynamo.

When we allow for $\mathcal{Q}^{R}=9.34 \mathrm{TW}$ of radiogenic heat in (4.10) and (4.21), we find that there is no longer a critical $\lambda$ for which $\mathcal{Q}^{D}$ is zero; now $\mathcal{Q}^{D}$ is always positive. The 
inequality (2.19) gives

$$
11.1 \mathrm{TW}<\mathcal{Q}_{\mathrm{CMB}}<14.7 \mathrm{TW}, \quad 5.3 \mathrm{Gyr}>\tau_{\mathrm{SIC}}>1.6 \mathrm{Gyr} .
$$

This range of $\tau_{\text {SIC }}$ includes the age of the Earth, i.e., it is possible that the SIC has existed over most of geological time and has produced a dynamo for which $1 \mathrm{TW}<\mathcal{Q}^{J}<2 \mathrm{TW}$. This is fortunate, because the thermodynamic obstacles to dynamo action in a totally fluid core $(\S 4.4)$ are not much eased by the radioactive sources. For such a core, the bounds (2.19) give

$$
12.9 \mathrm{TW}<\mathcal{Q}_{\mathrm{CMB}}<20.1 \mathrm{TW}, \quad 0.76 \mathrm{Gyr}>\tau_{\text {cooling }}>0.25 \mathrm{Gyr},
$$

which may be compared with (4.30). Moreover the core must rid itself of heat very rapidly, and the mantle must transmit it. The same is even true, to a lesser extent, when the inner core is present and (6.5) holds.

Let us suppose that the SIC has existed for 4 Gyr $(\lambda=10 / 3)$, and let us continue to suppose that $\mathcal{Q}^{R}$ is unchanging. We find that

$$
\mathcal{Q}_{\mathrm{CMB}}=11.6 \mathrm{TW}, \quad \mathcal{Q}^{D}=1.1 \mathrm{TW} .
$$

(If $\mathcal{Q}^{R}$ is halved, we find that, for $\tau_{\mathrm{SIC}}=4 \mathrm{Gyr}, \mathcal{Q}_{\mathrm{CMB}}=7.2 \mathrm{TW}$, as assumed by Glatzmaier and Roberts in their simulations; then $\mathcal{Q}^{D}=0.53 \mathrm{TW}$.) In terms of the efficiencies introduced in $\S 5,(6.8)$ gives

$$
\epsilon_{G} \approx 9 \%, \quad \epsilon_{D} \approx 0.3 \%, \quad f_{F} \approx 0.27
$$

The reduction of efficiency, as compared with (5.6) reflects the effect of the added thermal forcing created by $\mathcal{Q}^{R}$.

If we allow for the greater strength of the radioactive sources $4 \mathrm{Gyr}$ ago, we find that $\mathcal{Q}_{\mathrm{CMB}} \approx \mathcal{Q}^{R} \approx 82 \mathrm{TW}$ and $\mathcal{Q}^{D} \approx 11 \mathrm{TW}$ which, if the mantle is able to transmit so much heat, leaves abundent energy to drive a very strong dynamo. For such large values of $\mathcal{Q}^{R}$ both the pair of equations (4.24) and (4.25) and the pair (4.28) and (4.29) reduce approximately to

$$
\mathcal{Q}_{\mathrm{CMB}}=\mathcal{Q}^{R}, \quad \mathcal{Q}^{D}=0.12 \frac{T_{D}}{T_{\mathrm{CMB}}} \mathcal{Q}^{R},
$$

and the presence or absence of an inner core has no thermodynamic significance.

\section{CONCLUSIONS}

At first sight, it appears that the ohmic dissipation associated with the geomagnetic field is rather small in comparison with the thermal and gravitational energy sources available to drive the dynamo. As we saw in $\S 2.2$, less than $6 \mathrm{MW}$ of dissipation is the minimum sufficient to sustain the present dipole field of the Earth, but there is no less than $44 \mathrm{TW}$ of heat energy coming out of the Earth's surface. There is surely a huge "comfort-zone" here removing any worries that there is insufficient energy to drive the dynamo. Unfortunately, there are a large number of factors that chip away at this comfort-zone. First, the 6 
MW assumes the field is in an optimal configuration to reduce ohmic dissipation. A more realistic configuration, such as the interior field having the form of a free decay mode, increases the dissipation to around $80 \mathrm{MW}$. Recent simulations of the geodynamo suggest that the invisible component of the field which never leaves the core (consisting of both toroidal and poloidal components) is much larger than the $0.0005 \mathrm{~T}$ field escaping at the CMB. A toroidal field of 20 times this strength, $0.01 \mathrm{~T}$, is typical of what is found. This increases the dissipation by a factor of 400, and an additional factor of 2 is provided by the slightly higher dissipation rate of toroidal modes compared to poloidal modes. Thus the dissipation from the largest scale modes could well be around $0.08 \mathrm{TW}$.

The next factor to take into account is the contribution to ohmic dissipation from the smaller scale modes. As we argue in $\S 2$, the modes in the spherical harmonic range $n \sim 10-40$ are the most likely to be providing the bulk of the dissipation, although this depends on the form of the magnetic power spectrum. Not much is known about this at present, though it could be a fruitful field for further investigation. Our present estimates are based on the simulations of Roberts and Glatzmaier (2000a), and these suggest that the 0.08 TW large scale dissipation could be enhanced up to 1 or 2 TW by the smaller scale components. Thus we see that the $6 \mathrm{MW}$ "minimum" dissipation is a gross underestimate of the actual dissipation required to produce a $0.0005 \mathrm{~T}$ field coming out of the CMB. If viewed as a means of producing external dipole field, the dynamo process is extraordinarily inefficient, with most of the generated field never emerging at all, and with small scale "useless" components dissipating most of the magnetic energy.

There are still nevertheless $44 \mathrm{TW}$ of heat coming out of the Earth; surely this is sufficient to produce 1-2 TW of ohmic dissipation? There are two main problems here. First, on the traditional view, only about 7 TW (or may be less) of this 44 TW pass through the CMB. The rest is produced by radioactivity in the mantle, useless for the dynamo. The thermal heat flux is also subject to the "Carnot efficiency factor." Since the temperature difference between the CMB and the ICB is only around $20 \%$ of the actual temperature, only a small fraction of the available heat flux can go into ohmic dissipation. The gravitational energy associated with compositional convection is not subject to this efficiency factor tax, but unfortunately the amount of energy available from this source now appears to be less than originally thought, owing to new work on the crystallization process (§3.4). The net effect of these considerations is that it now appears that the energy budget for the geodynamo is rather tight, and we must start to consider ways in which either the process could be more efficient than we currently believe, or that there are new energy sources such as radioactivity in the core.

The energy budget calculations depend on a wide range of geophysical parameters, some of which are well-known, while others remain uncertain. We have revised the estimates given in Braginsky and Roberts (1995) in a number of ways. Their estimates were made using the PREM model, while here we have used the ak135 model of Kennett et al. (1995). The resulting differences are rather small, and do not affect the estimates of the various components of the energy and entropy fluxes very significantly. The heat flux conducted down the adiabat is significant in the core, so the energetics are sensitive to the values of the thermal conductivity assumed. These values are constantly revised as our knowledge of high pressure material physics improves, but the changes over the last 
six years have not led to very significant changes to the gross energy balance. The most significant uncertainties are connected with the freezing process and the growth of the inner core. The jump in density between the SIC and the FOC at the ICB boundary has been fairly well established by seismology, but this jump consists of two parts, one due to the contraction of iron on solidifying and one due to the release of light material. It now appears that the part due to contraction on solidification was previously underestimated, while correspondingly, the release of light material was overestimated. In consequence, the latent heat of crystallization has been revised upward (Anderson and Duba, 1997) leading to a significant increase in the estimate of total rate of latent heat release at the ICB, but the rate of gravitational energy release is now less than previously thought. Although the overall energy release is much the same as in the previous estimates, this change reduces the energy available for ohmic dissipation, because the Carnot efficiency factor bites into the extra thermal energy released by latent heat, but we lose the full amount of the decrease in gravitational energy. In consequence, if the slower estimates of core cooling are adopted, corresponding to the inner core being in existence for $3 \mathrm{Gyr}$, there is a danger that there is insufficient energy to drive a dynamo at all.

There is a particular problem with the early Earth. Paleomagnetic data reaching back 3 Gyr is of course far less certain than that accumulated for more recent epochs, but if the work of Kono and Tanaka (1995) is confirmed, dynamo theory will have to take on board the possibility that a geomagnetic field not dissimilar to the present field can be generated with no inner core. From the discussion in $\S 4.3$ we see that this makes the energy problem much more acute. To obtain a vigorous 1-2 TW dynamo with no inner core requires a great deal more heat flux through the CMB than the $7 \mathrm{TW}$ the current standard model suggests.

There are a number of ways in which the energy problem could be alleviated. Our knowledge of the composition of core material and of the high pressure physics that governs the behaviour near the ICB is still very incomplete, and it is possible that new investigations may increase estimates of gravitational release, alleviating the problem at least when there is a growing inner core. We should also remember that the field of dynamo simulation is still in its infancy, and it may be that more efficient dynamos emerge, allowing us to reduce the amount of ohmic dissipation while still maintaining an external field of the observed strength. This issue has not received very much attention to date, and further progress may well be possible. Whether new developments will improve the situation (or make it worse!) remains to be seen.

There is, however, another way of overcoming the difficulty if radioactivity is allowed in the core. There is then no problem in finding the energy required to drive a dynamo with or without an inner core. This may also have implications for other planetary dynamos, such as the recently discovered dynamo in Ganymede. In $\S 6$ we have presented the case for radioactive potassium entering the core via a nickel alloy. At present, our understanding of the planetary formation process is still very rudimentary; no doubt this important issue of the amount of radioactivity in planetary cores, which has profound implications for the thermal history of the solar system, will be further discussed by the geochemistry community. Another possible way of deciding this issue would be through mantle convection studies. The heat coming out of the core must pass through the thermal boundary layer 
at the base of the mantle, and then be convected up through the mantle. Observations of the thermal boundary layer, which is believed to be the $D^{\prime \prime}$-layer, or mantle convection simulations may be able to place useful constraints on the amount of heat going through the CMB.

Acknowledgments. One of us (PHR) was supported by the National Science Foundation with grant NSF EAR97-25627. A.C. was supported by a DOE Cooperative Agreement (\#DE-FCO8-98NU13410) between the High Pressure Science Center at the University of Nevada-Las Vegas, Department of Physics, and the US Department of Energy.

\section{REFERENCES}

Abramowitz, M. and Stegun, I.A. (eds.), Handbook of Mathematical Functions with Formulas, Graphs and Mathematical Tables, NBS Appl. Math. Ser., \#55 (1964).

Anderson, O.L. and Duba, A., "Experimental melting curve of iron revisited," J. Geophys. Res. 102, 22,659-22,669 (1997).

Backus, G.E., "Gross thermodynamics of heat engines in the deep interior of the Earth," Proc. Nat. Acad. Sci. Wash. 72, 1555-1558 (1975).

Backus, G., Parker, R. and Constable, C., Foundations of Geomagnetism, Cambridge UK: University Press (1996).

Boehler, R., "Temperatures in the Earth's core from melting point measurements of iron at high static pressures," Nature 363, 534-536 (1993).

Boehler, R., "Experimental constraints on melting conditions relevant to core formation," Geochim. Cosmochim. Acta 60, 1109-1112 (1996).

Boss, A.P., "Temperatures in protoplanetary Disks," Annu. Rev. Earth Planet. Sci. 26, 53-80 (1998).

Braginsky, S.I., "Magnetohydrodynamics of the Earth's core," Geomag. Aeron. 4, 898-916 (1964).

Braginsky, S.I. and Meytlis, V.P., "Local turbulence in the Earth's core," Geophys. Astrophys. Fluid Dynam. 55, 71-87 (1990).

Braginsky, S.I. and Roberts, P.H., "Equations governing Earth's core and the geodynamo," Geophys. Astrophys. Fluid Dynam., 79, 1-97 (1995).

Buffett, B.A., Huppert, H.E., Lister J.R. and Woods, A.W., "On the thermal evolution of the Earth's core," J. Geophys. Res. 101, 7989- 8006 (1996).

Bukowinski, M.S.T., "The Effect of pressure on the physics and chemistry of potassium," Geophys. Res. Letts. 3, 491-503 (1976).

Calderwood, A.R., "The Distribution of U, Th, and K in the Earth's Crust, Lithosphere, Mantle and Core: Constraints from an elemental Mass Balance Model and the Present day Heat flux". IN SEDI 2000, the 7th Symposium on Studies of the Earth's Deep Interior, Exeter, England, Abstract vol. S9.4 (2000).

Chabot, N.L. and Drake, M.J., "Potassium solubility in metal: The effects of composition at 15 kilobars and $1900 \mathrm{C}$ on partitioning between iron alloys and silicate melts," Earth Planet. Sci. Lett. 172, 323-335 (1999).

Davies, G.F., "Heat deposition and retention in a solid planet growing by impacts," Icarus 63, 45-68 (1985). 
Davies, G.F., "Heat and mass transport in the early Earth." IN: Origin of the Earth, Jones, J.H., and Newsom, H.E., eds., Oxford: University Press, New York, pp. 175194 (1990).

Dziewonski, A.M. and Anderson, D.L., "Preliminary reference Earth model," Phys. Earth Planet. Inter. 25, 297-356 (1981).

Glatzmaier, G.A. and Roberts, P.H., "A three-dimensional convective dynamo solution with rotating and finitely conducting inner core and mantle," Phys. Earth Planet. Inter. 91, 63-75 (1995a).

Glatzmaier, G.A. and Roberts, P.H., "A three-dimensional self-consistent computer simulation of a geomagnetic field reversal," Nature 377, 203-209 (1995b).

Glatzmaier, G.A. and Roberts, P.H., "An anelastic evolutionary geodynamo simulation driven by compositional and thermal convection," Physica, D 97, 81-94 (1996a).

Glatzmaier, G.A. and Roberts, P.H., "Rotation and magnetism of Earth's inner core," Science 274, 1887-1891 (1996b).

Glatzmaier, G.A. and Roberts, P.H., "Simulating the geodynamo", Contemp. Phys. 38, 269-288 (1997).

Glatzmaier, G.A., Coe, R.S., Hongre, L. and Roberts, P.H., "The role of the Earth's mantle in controlling the frequency of geomagnetic reversals", Nature 401, 885-890 (1999).

Goettel, K.A., "Partitioning of potassium between silicates and sulphide melts: experiments relevant to the Earth's core," Phys. Earth Planet. Inter. 6, 161-166 (1972).

Goettel, K.A., "Potassium in the Earth's core: evidence and implications". IN: Physics and Chemistry of Minerals and Rocks. Strews, R.G., jr. (ed.). John Wiley Interscience, pp. 479-489 (1974).

Goettel, K.A., "Models for the origin and composition of the Earth, and the hypothesis of Potassium in the Earth's core," Geophy. Surveys 2, 36-397 (1976).

Grevesse, N., Noels, A. and Sauval, A.J., "Standard abundances". IN: Cosmic Abundances, Holt, S.S., and Sonneborn, G., (eds.), Astronom. Soc. of the Pacific Conf. Ser. 99, 117-126 (1996).

Gubbins, D., "Observational constraints on the generation process of the Earth," Geophys. J. 47, 19-39 (1976).

Gubbins, D., "Energetics of the Earth's core," J. Geophys. 43, 453-464 (1977).

Gubbins, D., Masters, T.G. and Jacobs, J.A., "Thermal evolution of the Earth's core," Geophys. J. R. Astr. Soc. 59, 57-99 (1979).

Hart, S.R. and Zindler, A., "In search of a bulk Earth composition," Chem. Geol. 57, 247-267 (1986).

Hart, S.R. and Zindler, A., "Constraints on the nature and development of chemical heterogeneities in the mantle." IN: Mantle Convection, W.R. Peltier eds. Gordon and Breach Science Publishers, pp. 261-387 (1989).

Hewitt, J.M., McKenzie, D.P. and Weiss, N.O., "Dissipative heating in convective flows," J. Fluid Mech. 68, 721-738 (1975).

Humayun, M. and Clayton, R.N., "Precise determination of the isotopic composition of potassium: Application to terrestrial rocks and lunar soils," Geochim. Cosmochim. Acta 59, 2115-2130 (1995a). 
Humayun, M. and Clayton, R.N., "Potassium isotope cosmochemistry: genetic implications of volatile element depletion," Geochim. Cosmochim. Acta 59, 2131-2148 (1995b).

Ito, E., Morooka, K. and Ujike, O., "Dissolution of $\mathrm{K}$ in molten iron at high pressure and temperature," Geophys. Res. Letts. 20, 1651-1654 (1993).

Jeanloz, R., "The nature of the Earth's core," Annu. Rev. Earth Planet. Sci. 18, 357-386 (1990).

Jones, C.A. and Roberts, P.H., "Convection driven dynamos in a rotating plane layer", $J$. Fluid Mech. 404, 311-343 (2000).

Kennett, B.L.N., Engdahl, E.R. and Buland, R., "Constraints on seismic velocities in the Earth from traveltimes", Geophys. J. Int. 122, 108-124 (1995).

Kono, M. and Tanaka, H., "Intensity of the geomagnetic field in geological time: A statistical study." IN The Earth's Central Part: Its Structure and Dynamics Yukutake, T. ed., Terrapub, Tokyo, Japan, pp. 75-94 (1995).

Labrosse, S., Poirier, J.-P. and Mouël, J.-L., "On the cooling of Earth's core," Phys. Earth Planet. Inter. 99, 1-17 (1997).

Labrosse, S., Poirier, J.-P. and Mouël, J.-L., "The age of the inner core," to appear (2001).

Laio, A., Bernard, S., Chiarotti, G.L., Scandolo S. and Tosatti, E., "Physics of iron at Earth's core conditions," Science 287, 1027-1030 (2000).

Langel, R.A., "The main field". IN Geomagnetism 1, J.A. Jacobs (ed.), Academic Press, New York (1987).

Larimer, J.W., "The cosmochemical classification of the elements." IN: Meteorites and the Early Solar System. Kerridge, J.F., and Mathews, M.S. (eds.). University of Arizona Press, Tucson, Arizona, pp. 436-461 (1988).

Lewis, J.S., "Consequences of the presence of sulfur in the core of the Earth," Earth Planet. Sci. Letts. 11, 130-134 (1971).

Li, Jie, and Agee, C.B., "Geochemistry of mantle-core differentiation at high pressure," Nature 381, 687-689 (1996).

Li, Jie, and Agee, C.B., "Partitioning of volatile elements during core formation." IN Seventh Annual V.M. Goldschmidt Conference, LPI Contribution No. 921, Lunar and Planetary Institute, Houston, p. 126 (1997).

Liu, L.-G., "Potassium and the Earth's core", Geophys. Res. Letts. 13, 1145-1148 (1986).

Loper, D.E., "The gravitationally powered dynamo." Geophys. J. R. Astron. Soc. 54, 389-404 (1978).

Loper, D.E., "The nature and consequences of thermal interactions twixt core and mantle," J. Geomag. Geoelectr. 43, 79-91 (1991).

McDonough, W.F. and Sun, S.-S., "The composition of the Earth," Chem. Geol. 120, 223-253 (1995).

Merkel, S., Goncharov, A.F., Mao, H.-K., Gillet, Ph. and Hemley, R.J., "Raman spectroscopy of iron to 152 GigaPascals: Implications for Earth's inner core," Science 288, 1626-1629 (2000).

Miedema, A.R., de Chatel, P.F. and de Boer, F.R., "Cohesion in alloys - fundamentals of a semi-empirical model," Physica, B100, 1-28 (1980). 
Moffatt, H.K., Magnetic Field Generation in Electrically Conducting Fluids. Cambridge UK: University Press (1978).

Murthy, V.R., and Hall, H.T., "The chemical composition of the Earth's core: possibility of sulfur in the core," Phys. Earth Planet. Inter. 2, 276-282 (1970).

Murthy, V.R. and Hall, H.T., "The origin and chemical composition of the Earth's core," Phys. Earth Planet. Inter. 6, 123-130 (1972).

Ohtani, E., Yurimoto, H. and Seto, S., "Element partitioning between metallic liquid, silicate liquid, and lower mantle minerals: implications for core formation of the Earth," Phys. Earth Planet. Inter. 100, 97-114 (1997).

Palme, H., Larimer, J.W. and Lipshutz, M.E., "Moderately volatile elements." IN: Meteorites and the Early Solar System. Kerridge, J.F., and Mathews, M.S. (eds.). University of Arizona Press, Tucson, Arizona, pp. 436-461 (1988).

Parker, L.J., Atou, T. and Badding, J.V., "Transition element-like chemistry for potassium under pressure," Science 273, 95-97 (1996).

Poirier, J.-P. and Shankland, T.J., 1993. "Dislocation melting of iron and the temperature of the inner core," Geophys. J. Int. 115, 147-151.

Righter, K. and Drake, M.J., "Core formation in Earth's Moon, Mars, and Vesta," Icarus 124, 513-529 (1996).

Righter, K. and Drake, M.J., Metal-silicate equilibrium in a homogeneously accreting Earth: new results for Re," Earth Planet. Sci. Letts., 146 541-553 (1997a).

Righter, K. and Drake, M.J., "Prediction of siderophile element metal-silicate partition coefficients to $20 \mathrm{GPa}$ and $2800 \mathrm{oC}$ : the effects of pressure, temperature, oxygen fugacity, and silicate and metallic melt compositions," Phys. Earth Planet. Sci. Letts. 100, 115-134 (1997b).

Righter, K. and Drake, M.J., "Effect of water on metal-silicate partitioning of siderophile elements: a high pressure and temperature terrestrial magma ocean and core formation," Earth Planet. Sci. Letts. 171, 383-399 (1999).

Righter, K., Walker R.J. and Warren, P.H., "Significance of highly siderophile elements and osmium isotopes in the lunar and terrestrial mantles." IN: Origin of the Earth and Moon, Canup, R.M., Righter, K., eds., University of Arizona Press, pp. 291-322 (2000).

Ringwood, A.E., "On the chemical evolution and densities of the planets," Geochim. Cosmochim. Acta 51, 257-287 (1959).

Ringwood, A.E., Composition and Petrology of the Earth's Mantle. New York, McGraw Hill, 618 pp. (1975).

Ringwood, A.E., Origin of the Earth and Moon. New York: Springer (1979).

Roberts, P.H. and Glatzmaier, G.A., "A test of the frozen flux approximation using geodynamo simulations," Phil. Trans. R. Soc. London, A358 1109-1121 (2000a).

Roberts, P.H. and Glatzmaier, G.A., "Geodynamo theory and simulations," Rev. Mod. Phys. 72, 1081-1123 (2000b).

Sarson, G.R. and Jones, C.A., "A convection driven geodynamo reversal model," Phys. Earth planet. Inter. 111, 3-20 (1999).

Shannon, M.C. and Agee, C.B., "Percolation of core melts at lower mantle conditions," Science 280, 1059-1061 (1998). 
Shearer, P. and Masters, G., "The density and shear velocity contrast at the inner core boundary," Geophys. J. Int. 102, 491-496 (1991).

Sherman, D.M., "Chemical bonding and the incorporation of potassium into the Earth's core," Geophy. Res. Letts. 17, 693-696 (1990).

Somerville, M. and Ahrens, T.J., "Shock compression of KFeS2 and the question of potassium in the core," J. Geophy. Res. 85, 7016-7024 (1980).

St. Pierre, M. G., "On the local nature of turbulence in Earth's outer core," Geophys. Astrophys. Fluid Dynam. 83, 293-306 (1996).

Stacey, F.D., Physics of the Earth, Brisbane: Brookfield Press, $3^{\text {rd }}$ Edition (1992).

Stacey F.D. and Anderson, O.L., "Electrical and thermal conductivities of Fe-Ni-Si alloy under core conditions," Phys. Earth planet. Inter. (to appear) (2001).

Stevenson, D.J., Spohn, T. and Schubert, G., "Magnetism and thermal evolution of the terrestrial planets," Icarus 54, 466-489 (1983).

Sun, S.-S., "Chemical composition and origin of the Earth's primitive mantle," Geochim. Cosmochim. Acta 46, 179-192 (1982).

Suzuki, T., Akaogi, M. and Yagi, T., "Pressure dependence of Ni, Co and Mn partitioning between iron hydride and olivine, magnesiownstite and pyroxene," Phy. Earth Planet. Inter. 96, 209-220 (1996).

Thibault, Y. and Walter, M.J., "The influence of pressure and temperature on the metalsilicate partition coefficients of nickel and cobalt in a model $\mathrm{C} 1$ chondrite and implications for metal segregation in a deep magma ocean," Geochim. Cosmochim. Acta 59, 991-1002 (1995).

Wade, J. and Wood, B.J., "The Earth's 'missing' niobium may be in the core," Nature 409, 75-78 (2001).

Wanke, H., "Constitution of terrestrial planets," Phil. Trans. R. Soc. London, A303, 287302 (1981).

Wasson, J.T. and Kallemeyn, G.W., "Compositions of chondrites," Phil. Trans. R. Soc. London, A325, 535-544 (1988).

Williams, Q., Jeanloz, R., Bass, J., Svendsen, B. and Ahrens, T.J., "A melting curve for iron to 250 GigaPascals: a constraint on the temperature at Earth's center", Science 236, 181-182 (1987).

Winzenick, M., Vijayakumar, V. and Holzapfel, W.B., "High Pressure X-ray diffraction on potassium and rubidium up to 50 GPa," Phys. Rev., B50, 12,381-12,385 (1994). 
Table 1 . The effect of varying $\Delta^{s} V$

\begin{tabular}{|c|r|r|r|r|c|}
\hline $10^{6} \Delta^{s} V\left(\mathrm{~m}^{3} \mathrm{~kg}^{-1}\right)$ & 0 & 1 & $\mathbf{2}$ & 3 & 3.65 \\
\hline$\Delta^{s} \rho\left(\mathrm{kg} \mathrm{m}^{-3}\right)$ & 0 & 155 & $\mathbf{3 1 0}$ & 464 & 565 \\
$\Delta^{\xi} \rho\left(\mathrm{kg} \mathrm{m}^{-3}\right)$ & 565 & 410 & $\mathbf{2 5 5}$ & 101 & 0 \\
$r_{F S}$ & 0.47 & 0.34 & $\mathbf{0 . 2 1}$ & 0.083 & 0 \\
$\Delta \xi$ & 0.044 & 0.032 & $\mathbf{0 . 0 2 0}$ & 0.008 & 0 \\
$100 \Delta_{2}$ & 5.53 & 4.68 & $\mathbf{3 . 8 3}$ & 2.98 & 2.43 \\
$L\left(\mathrm{~J} \mathrm{~kg}{ }^{-1}\right)$ & 0 & 0.78 & $\mathbf{1 . 5 6}$ & 2.33 & 2.84 \\
$\mathcal{Q}^{L}(\mathrm{TW})$ & 0 & 2.00 & $\mathbf{4 . 0 0}$ & 6.00 & 7.30 \\
$\mathcal{Q}^{S}(\mathrm{TW})$ & 3.33 & 2.82 & $\mathbf{2 . 3 1}$ & 1.80 & 1.47 \\
$\mathcal{Q}^{G}(\mathrm{TW})$ & 1.13 & 0.82 & $\mathbf{0 . 5 1}$ & 0.20 & 0 \\
$E_{\lambda}(\mathrm{TW})$ & 4.45 & 5.63 & $\mathbf{6 . 8 2}$ & 7.99 & 8.76 \\
$D_{\lambda}(\mathrm{TW})$ & 1.53 & 1.61 & $\mathbf{1 . 6 9}$ & 1.77 & 1.82 \\
$\tau_{\text {SIC }}^{\text {crude }}(\mathrm{Gyr})$ & 2.9 & 3.1 & $\mathbf{3 . 3}$ & 3.4 & 3.5 \\
$\mathcal{Q}_{\text {CMB }}^{1}(\mathrm{TW})$ & 4.6 & 5.5 & $\mathbf{6 . 3}$ & 7.1 & 7.5 \\
$\tau_{\text {SIC }}^{1}(\mathrm{Gyr})$ & 1.22 & 1.29 & $\mathbf{1 . 3 4}$ & 1.41 & 1.45 \\
$\mathcal{Q}_{\mathrm{CMB}}^{2}(\mathrm{TW})$ & 7.2 & 8.6 & $\mathbf{9 . 9}$ & 11.0 & 11.7 \\
$\tau_{\text {SIC }}^{2}(\mathrm{Gyr})$ & 0.77 & 0.81 & $\mathbf{0 . 8 5}$ & 0.89 & 0.92 \\
$\mathcal{Q}_{\mathrm{c}}^{D}(\mathrm{TW})$ & 1.48 & 1.11 & $\mathbf{0 . 8 7}$ & 0.70 & 0.62 \\
$\tau_{\text {SIC }}^{\mathrm{c}}(\mathrm{Gyr})$ & 0.95 & 1.20 & $\mathbf{1 . 4 6}$ & 1.71 & 1.87 \\
\hline
\end{tabular}


Table 2. The effect of varying $T_{m}$

\begin{tabular}{|c|r|r|r|r|r|}
\hline$T_{m}\left({ }^{\circ} \mathrm{K}\right)$ & \multicolumn{1}{|c|}{0} & $\mathbf{3 0 0}$ & 700 & 1000 & 1500 \\
\hline$T_{\mathrm{ICB}}\left({ }^{\circ} \mathrm{K}\right)$ & 5400 & $\mathbf{5 1 0 0}$ & 4700 & 4400 & 3900 \\
$T_{\mathrm{CMB}}\left({ }^{\circ} \mathrm{K}\right)$ & 4182 & $\mathbf{3 9 5 0}$ & 3640 & 3408 & 3021 \\
$\bar{T}\left({ }^{\circ} \mathrm{K}\right)$ & 4753 & $\mathbf{4 4 8 8}$ & 4136 & 3872 & 3432 \\
$\mathcal{H}_{\mathrm{ICB}}(\mathrm{TW})$ & 0.29 & $\mathbf{0 . 2 6}$ & 0.22 & 0.19 & 0.15 \\
$\mathcal{H}_{\mathrm{CMB}}(\mathrm{TW})$ & 6.58 & $\mathbf{5 . 8 7}$ & 4.99 & 4.37 & 3.43 \\
$\Sigma\left(\mathrm{GW}^{\circ} \mathrm{K}^{-1}\right)$ & 0.183 & $\mathbf{0 . 1 7 2}$ & 0.159 & 0.149 & 0.132 \\
$L\left(\mathrm{~J} \mathrm{~kg}^{-1}\right)$ & 1.55 & $\mathbf{1 . 5 6}$ & 1.57 & 1.57 & 1.58 \\
$1^{100 \Delta_{2}}$ & 1.83 & $\mathbf{1 . 7 2}$ & 1.59 & 1.49 & 1.32 \\
$\mathcal{Q}^{L}(\mathrm{TW})$ & 3.99 & $\mathbf{4 . 0 0}$ & 4.02 & 4.04 & 4.06 \\
$\mathcal{Q}^{S}(\mathrm{TW})$ & 2.28 & $\mathbf{2 . 3 1}$ & 2.34 & 2.37 & 2.41 \\
$E_{\lambda}(\mathrm{TW})$ & 6.78 & $\mathbf{6 . 8 2}$ & 6.87 & 6.91 & 6.98 \\
$D_{0}(\mathrm{TW})$ & 0.70 & $\mathbf{0 . 6 2}$ & 0.53 & 0.46 & 0.36 \\
$D_{\lambda}(\mathrm{TW})$ & 1.68 & $\mathbf{1 . 6 9}$ & 1.70 & 1.70 & 1.71 \\
$\tau_{\text {SICde }}^{\text {crude }}(\mathrm{Gyr})$ & 2.9 & $\mathbf{3 . 3}$ & 3.8 & 4.4 & 5.6 \\
$\mathcal{Q}_{\mathrm{CMB}}^{1}(\mathrm{TW})$ & 6.6 & $\mathbf{6 . 3}$ & 5.9 & 5.6 & 5.2 \\
$\tau_{\mathrm{SIC}}^{1}(\mathrm{Gyr})$ & 1.28 & $\mathbf{1 . 3 4}$ & 1.44 & 1.52 & 1.65 \\
$\mathcal{Q}_{\mathrm{CMB}}^{2}(\mathrm{TW})$ & 10.2 & $\mathbf{9 . 9}$ & 9.5 & 9.2 & 8.8 \\
$\tau_{\text {SIC }}^{2}(\mathrm{Gyr})$ & 0.82 & $\mathbf{0 . 8 5}$ & 0.89 & 0.92 & 0.97 \\
$\mathcal{Q}_{\mathrm{c}}^{D}(\mathrm{TW})$ & 0.98 & $\mathbf{0 . 8 7}$ & 0.74 & 0.64 & 0.50 \\
$\tau_{\text {SIC }}^{\mathrm{c}}(\mathrm{Gyr})$ & 1.29 & $\mathbf{1 . 4 6}$ & 1.73 & 1.98 & 2.55 \\
\hline
\end{tabular}

Table 3. The effect of varying $\xi_{a}$

\begin{tabular}{|c|r|r|r|r|r|}
\hline$\xi_{a}$ & 0.03 & $\mathbf{0 . 0 9 5}$ & 0.12 & 0.16 & 0.2 \\
\hline$\Delta \xi$ & 0.011 & $\mathbf{0 . 0 2 0}$ & 0.025 & 0.034 & 0.042 \\
$\alpha^{\xi}$ & 1.90 & $\mathbf{1 . 0 0}$ & 0.79 & 0.59 & 0.48 \\
$100 \Delta_{2}$ & 3.26 & $\mathbf{3 . 8 3}$ & 4.15 & 4.65 & 5.16 \\
$\mathcal{Q}^{S}(\mathrm{TW})$ & 1.96 & $\mathbf{2 . 3 1}$ & 2.50 & 2.80 & 3.11 \\
$E_{\lambda}(\mathrm{TW})$ & 6.47 & $\mathbf{6 . 8 2}$ & 7.00 & 7.31 & 7.61 \\
$D_{\lambda}(\mathrm{TW})$ & 1.65 & $\mathbf{1 . 6 9}$ & 1.71 & 1.75 & 1.78 \\
$\tau_{\text {SIC }}^{\text {cude }}(\mathrm{Gyr})$ & 3.1 & $\mathbf{3 . 3}$ & 3.3 & 3.4 & 3.4 \\
$\mathcal{Q}_{\mathrm{CMB}}^{1}(\mathrm{TW})$ & 6.1 & $\mathbf{6 . 3}$ & 6.4 & 6.5 & 6.7 \\
$\tau_{\text {SIC }}^{1}(\mathrm{Gyr})$ & 1.30 & $\mathbf{1 . 3 4}$ & 1.37 & 1.40 & 1.42 \\
$\mathcal{Q}_{\mathrm{CMB}}^{2}(\mathrm{TW})$ & 9.5 & $\mathbf{9 . 9}$ & 10.0 & 10.2 & 10.4 \\
$\tau_{\text {SIC }}^{2}(\mathrm{Gyr})$ & 0.82 & $\mathbf{0 . 8 5}$ & 0.86 & 0.88 & 0.90 \\
$\mathcal{Q}_{\mathrm{c}}^{D}(\mathrm{TW})$ & 0.94 & $\mathbf{0 . 8 7}$ & 0.85 & 0.82 & 0.79 \\
$\tau_{\text {SIC }}^{\mathrm{c}}(\mathrm{Gyr})$ & 1.35 & $\mathbf{1 . 4 6}$ & 1.50 & 1.56 & 1.63 \\
\hline
\end{tabular}


Table 4. The effect of varying $h^{\xi}$

\begin{tabular}{|c|r|r|r|r|r|}
\hline$-10^{7} h^{\xi}\left(\mathrm{J} \mathrm{kg}^{-1}\right)$ & \multicolumn{1}{|c|}{0} & \multicolumn{1}{|c|}{1} & $\mathbf{1 . 6}$ & \multicolumn{1}{|c|}{2} & \multicolumn{1}{c|}{5} \\
\hline $100 \Delta_{2}$ & 2.63 & 3.38 & $\mathbf{3 . 8 3}$ & 4.13 & 6.39 \\
$\mathcal{Q}^{S}(\mathrm{TW})$ & 1.58 & 2.04 & $\mathbf{2 . 3 1}$ & 2.49 & 3.85 \\
$E_{\lambda}(\mathrm{TW})$ & 6.09 & 6.54 & $\mathbf{6 . 8 2}$ & 7.00 & 8.35 \\
$D_{\lambda}(\mathrm{TW})$ & 1.60 & 1.65 & $\mathbf{1 . 6 9}$ & 1.71 & 1.87 \\
$\tau_{\mathrm{SIC}}^{\text {crud }}(\mathrm{Gyr})$ & 3.1 & 3.2 & $\mathbf{3 . 3}$ & 3.3 & 3.6 \\
$\mathcal{Q}_{\mathrm{CMB}}^{1}(\mathrm{TW})$ & 6.0 & 6.2 & $\mathbf{6 . 3}$ & 6.4 & 7.0 \\
$\tau_{\text {SIC }}^{1}(\mathrm{Gyr})$ & 1.28 & 1.32 & $\mathbf{1 . 3 4}$ & 1.37 & 1.49 \\
$\mathcal{Q}_{\mathrm{CMB}}^{2}(\mathrm{TW})$ & 9.3 & 9.7 & $\mathbf{9 . 9}$ & 10.0 & 10.9 \\
$\tau_{\text {SIC }}^{2}(\mathrm{Gyr})$ & 0.81 & 0.83 & $\mathbf{0 . 8 5}$ & 0.86 & 0.94 \\
$\mathcal{Q}_{\mathrm{c}}^{D}(\mathrm{TW})$ & 0.97 & 0.91 & $\mathbf{0 . 8 7}$ & 0.85 & 0.72 \\
$\tau_{\text {SIC }}^{\mathrm{c}}(\mathrm{Gyr})$ & 1.30 & 1.40 & $\mathbf{1 . 4 6}$ & 1.50 & 1.78 \\
\hline
\end{tabular}

